\title{
Relativistic electrons in the outer radiation belt: Differentiating between acceleration mechanisms
}

\author{
Janet C. Green \\ Laboratory for Atmospheric and Space Physics, University of Colorado, Boulder, Colorado, USA
}

\author{
M. G. Kivelson \\ Institute of Geophysics and Planetary Physics and Department of Earth and Space Sciences, University of California, Los \\ Angeles, California, USA
}

Received 21 July 2003; revised 21 November 2003; accepted 22 January 2004; published 18 March 2004.

[1] Many theoretical models have been developed to explain the rapid acceleration to relativistic energies of electrons that form the Earth's radiation belts. However, after decades of research, none of these models has been unambiguously confirmed by comparison to observations. Proposed models can be separated into two types: internal and external source acceleration mechanisms. Internal source acceleration mechanisms accelerate electrons already present in the inner magnetosphere $(L<6.6)$, while external source acceleration mechanisms transport and accelerate a source population of electrons from the outer to the inner magnetosphere. In principle, the two types of acceleration mechanisms can be differentiated because they imply that different radial gradients of electron phase space density expressed as a function of the three adiabatic invariants will develop. Model predictions can be tested by transforming measured electron flux (given as a function of pitch angle, energy, and position) to phase space density as a function of the three invariants, $\mu, K$, and $\Phi$. The transformation requires adoption of a magnetic field model. Phase space density estimates have, in the past, produced contradictory results because of limited measurements and field model errors. In this study we greatly reduce the uncertainties of previous work and account for the contradictions. We use data principally from the Polar High Sensitivity Telescope energetic detector on the Polar spacecraft and the Tsyganenko and Stern [1996] field model to obtain phase space density. We show how imperfect magnetic field models produce phase space density errors and explore how those errors modify interpretations. On the basis of the analysis we conclude that the data are best explained by models that require acceleration of an internal source of electrons near $L^{*} \sim 5$. We also suggest that outward radial diffusion from a phase space density peak near $L^{*} \sim 5$ can explain the observed correspondence between flux enhancements at geostationary orbit and increases in ULF wave power. INDEX TERMS: 2720 Magnetospheric Physics: Energetic particles, trapped; 2788 Magnetospheric Physics: Storms and substorms; 2772 Magnetospheric Physics: Plasma waves and instabilities; 2752 Magnetospheric Physics: MHD waves and instabilities; 2778 Magnetospheric Physics: Ring current; KEYWORDS: geomagnetic storm, phase space density, relativistic electrons

Citation: Green, J. C., and M. G. Kivelson (2004), Relativistic electrons in the outer radiation belt: Differentiating between acceleration mechanisms, J. Geophys. Res., 109, A03213, doi:10.1029/2003JA010153.

\section{Introduction}

[2] On 31 January 1958 the Explorer I spacecraft was launched into Earth orbit carrying a cosmic ray experiment designed by Dr. James Van Allen [Van Allen et al., 1959; Van Allen and Frank, 1959]. Although it was intended to measure cosmic rays, the experiment was also sensitive to highly energetic electron flux. The measurements revealed that high fluxes of relativistic electrons encircle the Earth in what is now known as the Van Allen radiation belts. Since

Copyright 2004 by the American Geophysical Union. 0148-0227/04/2003JA010153\$09.00
Van Allen's unanticipated discovery, researchers have observed dramatic relativistic electron flux increases, often during geomagnetic storms, and have sought to identify acceleration mechanisms capable of producing these rapid flux increases.

[3] Many acceleration mechanisms have been proposed. They include variations on two themes: acceleration by radial diffusion from a source at and beyond geostationary orbit which we refer to as external source acceleration mechanisms [e.g., Elkington et al., 1999; Hudson et al., 2000; Kim et al., 2000; Li et al., 2001; Elkington et al., 2003] and in situ acceleration at $L<6.6$ by wave particle interactions, referred to here as internal source acceleration 
mechanisms [e.g., Albert, 2000; Liu et al., 1999; Summers et al., 1998; Summers and Ma, 2000]. None of these models has been unambiguously confirmed by comparison with observations. The details of individual models will not be discussed here but can be found in reviews by Friedel et al. [2002] and O'Brien et al. [2003].

[4] External source acceleration mechanisms rely on radial diffusion to transport and accelerate a source population of electrons from the outer to the inner magnetosphere. The transportlacceleration process violates the electrons' third adiabatic invariant while conserving the first two. Internal source acceleration mechanisms locally accelerate a population of electrons already present in the inner magnetosphere. These mechanisms violate either the first or second invariant.

[5] The goal of this paper is not to identify a specific acceleration mechanism but to determine which type of mechanism, external or internal, is principally responsible for accelerating electrons by comparing predicted signatures of radial gradients to measured data. Previous studies with similar goals have given ambiguous and sometimes conflicting results. Here we explain how the data can be used to differentiate between the two categories. We consider the limitations of previous studies that attempted to do so, and show how the current study addresses the uncertainties of previous work.

\subsection{Differentiating Between Internal and External Acceleration Mechanisms}

[6] The types of acceleration mechanisms that we have described predict different radial gradients of electron phase space density calculated as a function of the three adiabatic invariants, $f\left(\mu, K, L^{*}\right)$, where $\mu$ is the first invariant, $K$ is the second invariant and $L^{*}$ represents the third invariant (For a complete definition of the invariants see Appendix A). Electron phase space density is a quantity useful for theoretical analyses but it is not directly measured. Particle detectors typically measure electron flux, $j(E, \alpha, \vec{x})$, as a function of energy $(E)$, pitch angle $(\alpha)$, and spacecraft position $(\vec{x})$. Therefore, before comparing observations and predictions the data must be transformed from measured electron $j(E, \alpha, \vec{x})$ to $f\left(\mu, K, L^{*}\right)$.

[7] External source acceleration models rely on radial diffusion to transport and accelerate electrons. Radial diffusion is a process whereby time varying magnetic and electric fields perturb the radial position of an electron causing random walk motion and diffusive transport in much the same way that Brownian motion leads to diffusion in a gas [Falthammer, 1965, 1966; Schulz and Lanzerotti, 1974; Elkington et al., 1999, 2003]. The radial diffusion equation,

$$
\frac{\partial f\left(\mu, K, L^{*}\right)}{\partial t}=\left(\frac{\partial D}{\partial L^{*}}-\frac{2 D}{L^{*}}\right) \frac{\partial f}{\partial L^{*}}+D \frac{\partial^{2} f}{\partial L^{* 2}}
$$

describes the time variation of phase space density at a given $L^{*}$ and constrains gradients produced by external source acceleration mechanisms. The diffusion rate is set by the coefficient $D$ which is a function of $L^{*}$ as well as the amplitude of magnetic and electric field perturbations and can take various forms [e.g., Elkington et al., 2003]. The diffusion equation demonstrates how radial diffusion

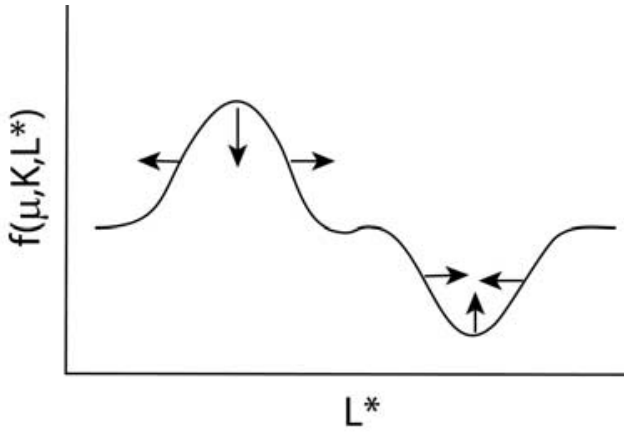

Figure 1. Schematic showing how radial diffusion reduces phase space density gradients.

reduces gradients. If local phase space density maxima (where $\partial^{2} f / \partial L^{*^{2}}<0$ and $\partial f / \partial L^{*}=0$ ) develop at low $L^{*}$, the peak will decrease in time as electrons are transported away. Local minima, on the other hand, increase as electrons move radially inward as illustrated schematically in Figure 1 [Walt, 1994]. Thus external source acceleration mechanisms cannot produce phase space density peaks or negative gradients inward of the electron source region.

[8] The diffusion coefficient in the radiation belts is thought to be of the form $D=D_{0} L^{n}$ [e.g., Schulz and Lanzerotti, 1974] where $D_{0}$ is related to wave power and $n$ ranges from 6 to 11. Figure 2a shows schematically how radial diffusion from a source outside of geosynchronous changes the phase space density versus $L^{*}$ profile during an acceleration event assuming $D \sim L^{* 10}$. The electron acceleration event begins with a positive phase space density slope (shaded trace). Electrons are transported inward and the phase space density increases at all $L^{*}<8$ until it reaches a steady state where $d f / d t=0$ (black trace).

[9] Figure 2b shows how the phase space density of electrons with fixed $\mu$ and $K$ evolves for internal source acceleration mechanisms. Internal mechanisms locally accelerate electrons (near $L^{*}=4$ in this example) forming a phase space density peak in the inner magnetosphere. Radial diffusion both inward and outward transports electrons away from the source region at low $L^{*}$ increasing the phase space density even where the acceleration mechanism is not active. The shaded trace in Figure $2 \mathrm{~b}$ shows the beginning of a phase space density peak formed by a local acceleration mechanism. The black trace shows the evolution of phase space density as the peak increases with time and electrons diffuse radially away from the source at low $L^{*}$.

[10] Differentiating between the two mechanisms seems a simple task of identifying peaks in the phase space density versus $L^{*}$ profile. Phase space density peaks at low $L^{*}$ would confirm acceleration of an internal source of electrons whereas a phase space density maximum at large $L^{*}$ would imply acceleration by radial diffusion from an external source. However, some care must be taken when identifying peaks. Selesnick and Blake [2000] demonstrated that local phase space density peaks do not always indicate internal acceleration. Electron acceleration by radial diffusion from an external source combined with a changing source population or other loss mechanism at large $L^{*}$ can also produce local peaks as illustrated by Figure 3 . The 


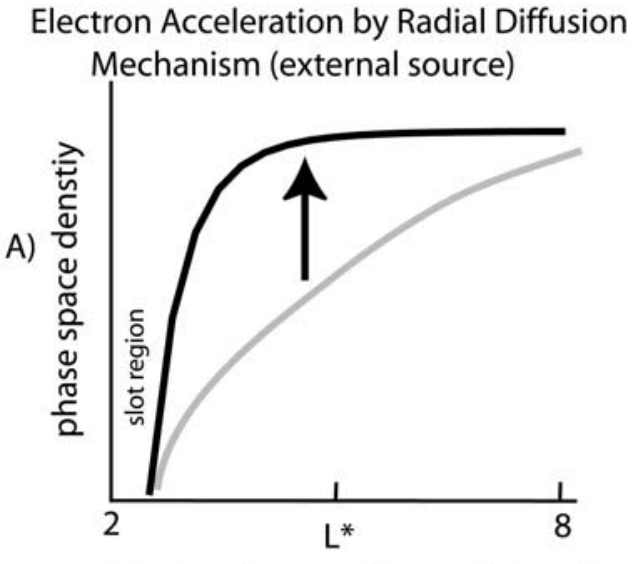

Electron Acceleration by Internal Source Mechanism

B)

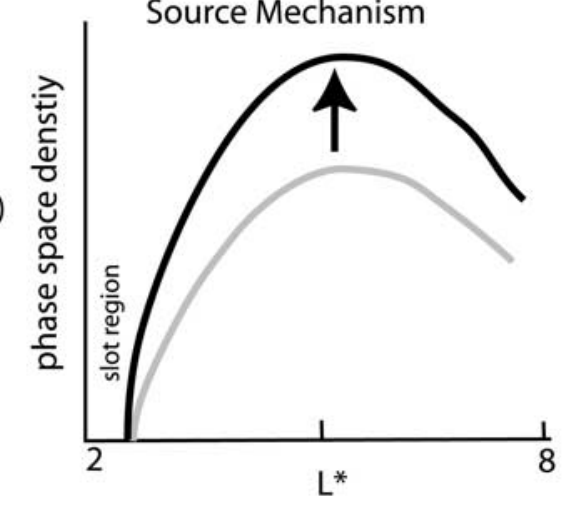

Figure 2. Schematic comparing the type of phase space density increases produced by radial diffusion from an external source and local acceleration of an internal electron source. (a) A phase space density increase caused by radial diffusion from an external source. The shaded trace represents phase space density at the start of an acceleration event. The black trace shows the phase space density once equilibrium is achieved. The black trace is obtained by solving the radial diffusion equation with $\partial f / \partial t=0$, where $f$ is phase space density. The solution assumes an inner boundary condition of zero (representing the slot region) and diffusion coefficient $D \sim L^{* 10}$. (b) Phase space density increases predicted by local internal source acceleration mechanisms. The shaded trace represents the phase space density at the start of an acceleration event. The black trace shows the phase space density after the electron acceleration.

schematic shows an initial phase space density versus $L^{*}$ profile consistent with acceleration by radial diffusion from an external source (black trace). After the initial phase space density increase, losses at large $L^{*}$ produce a profile with a peak at low $L^{*}$ (shaded trace). Whereas this scenario creates a local phase space density peak, such a peak is readily distinguished from one caused by internal source acceleration by tracking the radial dependence of phase space density versus time during the entire acceleration interval. As explained previously, radial diffusion from a source at large $L^{*}$ cannot produce a peak at low $L^{*}$ that increases with time.

[11] A note of caution must be added. The evidence distinguishing internal and external acceleration mechanisms relies on a derived quantity $f\left(\mu, K, L^{*}\right)$ that is subject to potentially large errors. To elaborate, specifying $f(\mu, K$, $\left.L^{*}\right)$ requires calculation of the adiabatic invariants. Errors are introduced because invariant calculations rely on assumptions and models rather than measured quantities. For example, calculating the first invariant, $\mu$, requires specification of the local magnetic field, particle energy, and pitch angle. In some situations these measurements are not all available. Thus a model magnetic field and/or an assumed pitch angle distribution are substituted for measurements. Calculating the second and third invariant, which correspond to particle bounce and drift motion, requires knowledge of the global configuration of the magnetic field that no single spacecraft measurement can provide. A global magnetic field model is always invoked to calculate these two invariants. Quantifying how assumptions and imperfections in the chosen field model add to create errors in invariant calculations and ultimately the phase space density estimates is challenging. Yet it is critical to establish whether possible sources of error affect the radial gradients before drawing conclusions. We discuss sources of error at length later in the paper.

\subsection{Previous Studies}

[12] Our study is not the first to examine phase space density gradients in an effort to understand relativistic electron acceleration. Several important studies laid the ground work by developing methods for calculating phase space density from measured particle fluxes and comparing the gradients to the model predictions outlined above. However, these studies in some cases gave contradictory results because they relied on data with inadequate temporal resolution and limited spatial and pitch angle coverage. Most importantly these studies did not estimate phase space density errors introduced by relying on imperfect magnetic field models. Here we discuss the results and limitations of three previous studies to highlight the improvements provided by our current work.

[13] Hilmer et al. [2000] used GPS and LANL electron data and found radial gradients of phase space density consistent with external source acceleration mechanisms. They compared electron phase space density measured by

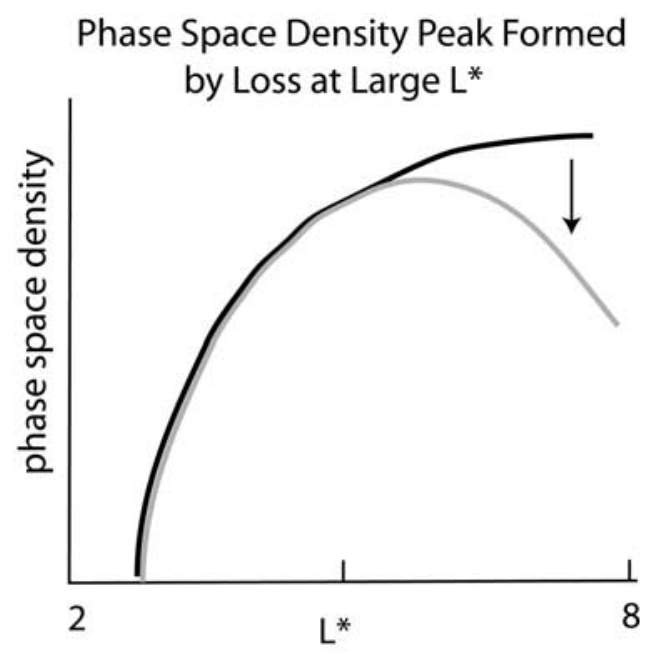

Figure 3. Schematic showing how losses at large $L^{*}$ cause a peak in the phase space density versus $L^{*}$ profile. 
GPS satellites at $L=4.2$ to electron phase space density at $L=6.6$ measured by LANL satellites. They considered only data measured at the equator corresponding to electrons with $K=0$ and $\mu=2100 \mathrm{MeV} / \mathrm{G}$. They examined 31 storms and found higher phase space density at $L=6.6$ than at 4.2 in 27 of them. The results are consistent with transport and acceleration of electrons from a source outside of $L=6.6$. However, neither magnetic field nor pitch angle measurements were available, and the estimation of the missing data introduced phase space density errors of uncertain magnitude. In particular, both GPS and LANL detectors are nearly omnidirectional and provide no pitch angle information. Therefore the Hilmer et al. [2000] study assumed the spin averaged flux equaled the flux of electrons with $90^{\circ}$ equatorial pitch angle and $K=0$. This assumption may not be valid during storm times when electron pitch angles change and show significant local time differences [Selesnick and Blake, 2002]. A dipolar magnetic field was used to calculate the energy of electrons with constant $\mu$ but the magnetic field changes markedly during storms and the change calls this assumption into question. Most importantly, because of the limited radial extent of the satellite orbits, the phase space density gradient was determined from only two point measurements at $L=4.2$ and $L=6.6$. Any phase space density peaks between these radial distances would not be detected.

[14] Brautigam and Albert [2000] calculated electron phase space density from CRRES MEA data during one storm and, unlike Hilmer et al. [2000], found radial gradients consistent with internal source acceleration. Brautigam and Albert [2000] compared electron phase space density versus $L^{*}$ profiles to a radial diffusion simulation with time-dependent outer boundary conditions. The study analyzed electrons with small second invariants $\left(J=1.78 \times 10^{-16} \mathrm{~g}(\mathrm{~cm} / \mathrm{s}) \mathrm{R}_{\mathrm{E}}\right)$ and $\mu$ values ranging from 100 to $1000 \mathrm{MeV} / \mathrm{G}$ and compared phase space density 8 days after the storm main phase to that predicted by the diffusion simulation. The phase space density of electrons with $\mu<316 \mathrm{MeV} / \mathrm{G}$ agreed well with the simulation predictions while the phase space density of electrons with $\mu>316 \mathrm{MeV} / \mathrm{G}$ did not. At these high $\mu$ values, the simulation produced positive phase space density gradients. The data, on the other hand showed negative phase space density gradients outside of $L^{*}=4.5$ indicative of internal source acceleration. However, the study did not clearly differentiate between phase space density peak increases such as those shown in Figure $2 \mathrm{~b}$ and peaks formed from losses at large $L^{*}$ such as those shown in Figure 3.

[15] Selesnick and Blake [2000] calculated electron phase space density using data obtained by the Polar High Sensitivity Telescope (HIST) for 2 months, January 1998 and May 1998, using the IGRF [Barton, 1997], Olsen-Pfitzer [Olson, 1974], T89 [Tsyganenko, 1989], and T96 [Tsyganenko and Stern, 1996] magnetic field models. They showed that phase space density gradients were highly dependent on the magnetic field model used. For example, no clear phase space density peaks developed in the May 1998 storm when $f(\mu, K$, $L^{*}$ ) was obtained using the T96 and Olsen-Pfitzer field models. Yet when $f\left(\mu, K, L^{*}\right)$ was evaluated using the IGRF model, a peak appeared near $L^{*} \sim 5$. The study demonstrated that phase space density estimates and gradients are strongly affected by the choice of field model, but did not examine just why the field models gave different results. The study concluded that the T89 model gave the best inbound/outbound (day/night) agreement and suggested a source in the range $4<L^{*}<6$ but that improved magnetic field models were required for confirmation.

\subsection{Goal of Current Work}

[16] The goal of this study, as in the past work described above, is to differentiate between internal and external source electron acceleration models by examining the temporal evolution of electron phase space density gradients during geomagnetic storms. We calculate electron phase space density using the Polar HIST and Magnetic Field Experiment (MFE) data that provide required pitch angle and local magnetic field information with good radial and time coverage. Most importantly, we show how imperfections in the unmeasured quantity, the assumed magnetic field model, produce phase space density errors and we explore how those errors modify key aspects of the phase space density estimates. The transformation method and associated errors are described in section 2. Section 3 evaluates the phase space density gradients that develop during two electron acceleration events and includes error analysis. Section 4 discusses the implications of the results for acceleration models. We conclude in section 5 that the data are best explained by models with localized acceleration of electrons occurring inside of geostationary orbit.

\section{Instrumentation, Phase Space Density Calculations, and Error Analysis}

\subsection{Instrumentation}

[17] The electron fluxes used in this study were obtained by the HIST instrument onboard the Polar spacecraft [Blake et al., 1995]. The instrument measures relativistic electron flux at energies between 0.7 and $7 \mathrm{MeV}$. As the spacecraft spins, data are collected in 16 different look directions each spanning $22.5^{\circ}$, giving full pitch angle coverage. The instrument calibration is described by Selesnick and Blake [2000] and Contos [1997]. Through much of the mission the HIST instrument accurately measures electron flux but during periods of high count the data are anomalous as a result of instrument saturation. During periods of exceptionally high fluxes, count rates are assumed to represent a lower limit. Throughout the paper this anomalous data will be plotted as shading. A more detailed explanation and discussion of approaches used to identify questionable intervals is given by Green [2002].

[18] The Polar MFE data [Russell et al., 1995] that provides the local vector magnetic field are used to transform the HIST electron flux into phase space density as a function of the adiabatic invariants. The MFE data is required to determine the pitch angles and $\mu$ values of the HIST data and to test the validity of the model field. Ancillary parameters are taken from the omniweb data set which provides measured solar wind parameters as well as geomagnetic indices. The omniweb data is used as input to the T96 field model as described in more detail below.

\subsection{Phase Space Density Calculations}

[19] We calculate phase space density using a similar technique to that of Green and Kivelson [2001]. The 
measured $j(E, \alpha, \vec{x}, t)$, is converted to $f\left(\mu, K, L^{*}, t\right)$, by selecting energies and pitch angles that correspond to particular values of $\mu, K$, and $L^{*}$ (For complete definitions of the adiabatic invariants see Appendix A). Four steps in the procedure are outlined below.

\subsubsection{Step 1}

[20] Change flux $j(E, \alpha, \vec{x}, t)$ as a function of energy, pitch angle, position, and time to phase space density, $f(E, \alpha, \vec{x}, t)$. The HIST data set gives $j(E, \alpha, \vec{x}, t)$ measured at 14 energies and 16 pitch angles. Phase space density is obtained from $f(E, \alpha, \vec{x}, t)=j(E, \alpha, \vec{x}, t) / p^{2}$ using $p^{2}=\left(E^{2}+2 m_{0} c^{2} E\right) / c^{2}$ where $p$ is the relativistic momentum, $c$ is the speed of light and $m_{0}$ is the electron rest mass [Schulz and Lanzerotti, 1974]. (Note that $E$ is the kinetic energy and not the total energy $E+m_{0} c^{2}$.)

\subsubsection{Step 2}

[21] Obtain $K(\alpha, \vec{x}, t)$ and correspondingly $\alpha(K, \vec{x}, t)$ and thereby obtain $f(E, K, \vec{x}, t)$. Relevant data are restricted to pitch angles corresponding to fixed $\mathrm{K}$. To find the pitch angles corresponding to fixed $K$, we calculate $K$ values for local pitch angles from $5^{\circ}$ to $90^{\circ}$ at $5^{\circ}$ intervals and $20 \mathrm{~s}$ time steps along the orbit. We calculate $K$ using the T96 field model [Tsyganenko and Stern, 1996] and the UNILIB code provided by the Belgian Institute for Space Aeronomy (BISA) available on the Web at http://www.magnet.oma.be/ home/unilib. We interpolate between the $18 \mathrm{~K}$ values corresponding to local pitch angles between $5^{\circ}$ and $90^{\circ}$ to find the pitch angle corresponding to any desired $K$.

[22] The T96 model used in the calculations requires as input parameters: the solar wind $B_{y}, B_{z}$, the dynamic pressure, and Dst. We use the omni data set as input to the model. The model is only valid for a restricted range of solar wind and $D s t$ values. These ranges are 0.5 to $10 \mathrm{nPa}$ for dynamic pressure, -100 to $20 \mathrm{nT}$ for $D s t,-10$ to $10 \mathrm{nT}$ for IMF $B_{y}$ and -10 to $10 \mathrm{nT}$ for IMF $B_{z}$. The measured solar wind or Dst values may fall outside these limits especially during the main phase of a storm. When this happens we use the model corresponding to the maximum or minimum allowed value instead of the measured value.

[23] We fit the pitch angle distributions at each of the 14 energy channels to the following function,

$$
j(\alpha)=C_{0} \sin \alpha+C_{1} \sin ^{C_{2}} \alpha
$$

using the Levenberg-Marquardt nonlinear least squares method [Press et al., 1988]. This form was used because it fits both butterfly and highly peaked distributions. The data have now been expressed as phase space density at fixed $K$, still measured at 14 energies.

\subsubsection{Step 3}

[24] Express $E$ as a function of $\mu, K$ position and time and thereby phase space density $f(\mu, K, \vec{x}, t)$. Relevant data are restricted to the energy corresponding to fixed $\mu$. The energy at constant $\mu$ is found using the definition of $\mu$ to solve for the kinetic energy $E$.

$$
p^{2}=\frac{2 m_{0} B \mu}{\sin ^{2} \alpha_{k}} \quad p^{2}=\left(E^{2}+2 m_{0} c^{2} E\right) / c^{2}
$$

Here $\alpha_{k}$ is the pitch angle corresponding to the value of $K$ found in step 2 . The magnetic field strength, $B$, is obtained from the MFE instrument. The phase space density at the energy corresponding to fixed $\mu$ is found by fitting $f(E, K, \vec{x}$, $t$ ) versus the 14 measured energies to an exponential.

\subsubsection{Step 4}

[25] Change phase space density as a function of $\mu, K$, and position to phase space density as a function of $\mu, K$, and $L^{*} . L^{*}$ is determined by tracing the electron drift in a model magnetic field and calculating the magnetic flux enclosed. Electron drift paths are slightly dependent on the pitch angle of the particle. We use the pitch angle corresponding to the desired value of $K$. As with the second invariant calculations we use the T96 model and the BISA UNILIB code. After computing the magnetic flux enclosed within the drift orbit, we assign $L^{*}$ by identifying the equatorial radial distance (in $\mathrm{Re}$ ) that encloses the same amount of flux in a dipolar magnetic field.

\subsection{Errors}

[26] Differentiating between the two categories of acceleration mechanisms depends critically on accurately obtaining the radial profile of phase space density. Here we focus on how errors may affect the calculated radial profile. More specifically, we are interested in whether errors may change an outward radial phase space density gradient indicative of radial diffusion from an external source population to one with a peak at low $L^{*}$ indicative of an internal source acceleration mechanism.

[27] Errors in our calculation of phase space density arise from two sources: poor data fits and imperfect magnetic field models. Poor data fits (step 2 and step 3) can occur if our chosen functional forms do not accurately represent the data or uncertainties in the measurements become large because of low count rates. Imperfect magnetic field models result in inaccurate estimates of $L^{*}$ and $K$. We explore the significance of each of these errors and develop analysis methods to be applied to the two example acceleration events described later in section 3 .

\subsubsection{Errors of Poor Fits}

[28] Steps 2 and 3 of the phase space density calculation require fitting the measured data to functional forms. In step 2 we fit the pitch angle distribution for each energy channel to a functional form and use a bootstrap method with replacement to define the $95 \%$ confidence range of the fits at the pitch angle of constant $K$. More precisely, we fit 32 random samples of the pitch angle distribution. The $95 \%$ confidence level is the range within which $95 \%$ of these fits lie. We use these confidence ranges to do a weighted fit of $f(E, K, \vec{x}, t)$ versus $E$ in step 3 . The bootstrap method is used again to define the $95 \%$ confidence range of the energy distribution fits at the energy of constant $\mu$. These errors can be accurately quantified and are not large enough to significantly affect the radial profile of phase space density as will be demonstrated later in the analysis of two example events.

\subsubsection{Errors in Determination of $L^{*}$}

[29] $L^{*}$ depends inversely on $\Phi$, the total magnetic flux enclosed by the drift orbit of an electron, and so is affected by the global accuracy of the magnetic field model. An imperfect field model will change $L^{*}$ in predictable ways. If the field model underestimates $\Phi$, the calculated $L^{*}$ will be larger than expected. Likewise, if the field model overestimates $\Phi, L^{*}$ will be smaller than expected. Therefore, 


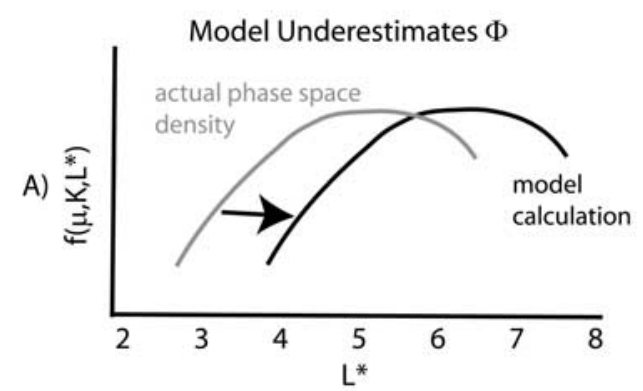

Model Overestimates $\Phi$
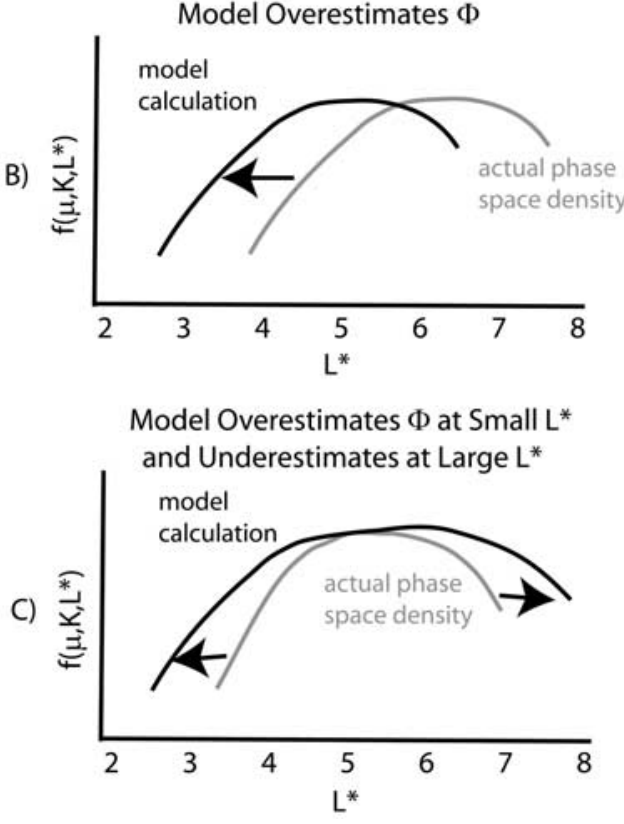

Figure 4. Schematic showing how an error in the calculation of the electrons third invariant $\Phi$ will affect the profile of phase space density versus $L^{*}$. (a) Underestimating $\Phi$ shifts the phase space density trace to larger $L^{*}$. (b) Overestimating $\Phi$ shifts the phase space density trace to smaller $L^{*}$. (c) Overestimating $\Phi$ at low $L^{*}$ and underestimating at large $L^{*}$ stretches the phase space density versus $\mathrm{L}^{*}$ profile.

errors in $L^{*}$ shift the calculated phase space density radially as shown by the cartoon of Figure 4 .

[30] The cartoon depicts three scenarios. In the first scenario, the field model systematically underestimates $\Phi$ and the calculated phase space density versus $L^{*}$ profile shifts to larger $L^{*}$. In the second scenario, the field model systematically overestimates $\Phi$ and the calculated phase space density versus $L^{*}$ profile shifts to smaller $L^{*}$. In the third scenario the model overestimates $\Phi$ at small $L^{*}$ and underestimates $\Phi$ at large $L^{*}$ causing the phase space density versus $L^{*}$ profile to stretch. Inaccurate calculation of $L^{*}$ does not change the value of phase space density. The errors only shift the value radially. These errors will not change the radial dependence of phase space density from one with an outward gradient to one with a peak at low $L^{*}$ and can be ignored as we seek to distinguish between the two acceleration mechanisms.

\subsubsection{Errors in Determination of $\boldsymbol{K}$}

[31] Calculating $K$ requires integrating the magnetic field along the bounce path of an electron between its mirror points. The integral depends on the length of the field line and the strength of the magnetic field along it. These values are provided by a magnetic field model. Errors in $K$ arise from imperfections in the magnetic field model. An error in the phase space density estimate occurs because the inaccurate estimate of $K$ changes the pitch angle used in step 2. The way in which erroneous estimates of $K$ affect phase space density calculations is best illustrated by an example such as shown in Figure 5. Figure 5a is a schematic showing phase space density at constant $\mu$ as a function of $K$. Phase space density typically decreases as $K$ increases as shown. The decreasing profile occurs because large $K$ values correspond to electrons with small pitch angles that travel far along the field line. Typical pitch angle distributions show that flux and phase space density decrease as pitch angle decreases. In addition, electrons with small pitch angles correspond to higher energies than electrons with large pitch angles when both have the same $\mu$ value. If the model overestimates $K$ values, the phase space density trace shifts to the right to larger $K$. The phase space density of a chosen fixed $K$ will be overestimated. Likewise, if the model underestimates $K$ the phase space density trace shifts left as in Figure $5 \mathrm{~b}$. The phase space density for fixed $K$ will be underestimated. $K$ increases as the length of the field line increases. Thus $K$ and phase space density will be overestimated when the stretching of the model is too large. Likewise $K$ and phase space density will be underestimated when the stretching is too small.
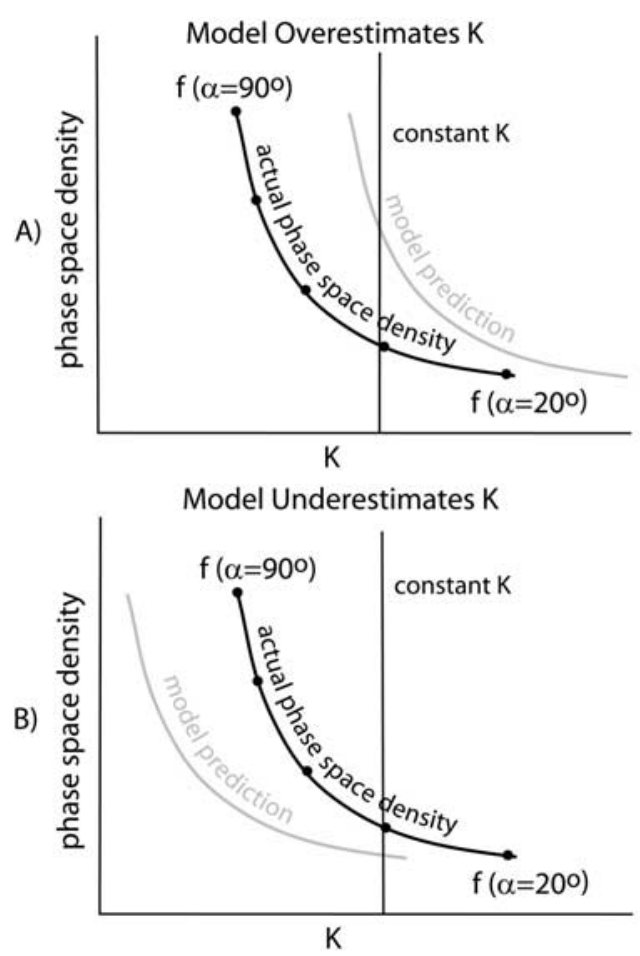

Figure 5. Schematic showing how an error in the calculation of the electron's second invariant, $K$, affects phase space density. (a) Overestimating $K$ values causes an overestimate of phase space density at constant $K$. (b) Likewise, underestimating $K$ results in an underestimate of phase space density at constant $K$. 
[32] The qualitative illustration shows that an error in the estimate of $K$ introduces an error in the estimate of phase space density which can affect the radial profile. The challenge is to quantify those errors. Unfortunately, stringent error analysis methods cannot be applied to this problem. For example, if the error in the magnetic field model were known at every point in space it could be propagated through the integral in the calculation of $K$ to determine a value $K \pm \Delta K$ and $f \pm \Delta f$ where $\Delta K$ and $\Delta f$ are the errors. However, the inaccuracy in the magnetic field model is not quantified. Instead, we estimate phase space density errors by testing the sensitivity of our estimates to changes in the magnetic field model by using a range of magnetic field models from a stretched, more geomagnetically active field to a quiet time, more dipolar field model. Using a stretched field model overestimates $K$ and phase space density. The less stretched field model underestimates the $K$ and phase space density. We generate a range of field models and phase space density estimates by modifying the inputs to the T96 field model.

[33] Intuition alone does not reveal how modifying each input parameter will affect the stretching of the model magnetic field and our estimates of $K$ and phase space density. To test the influence of each parameter we calculate pitch angles corresponding to constant $K$ at various points in the magnetosphere while modifying the input parameters individually. The test shows that the input Dst value most affects the calculation [Green, 2002]. The influence of the Dst parameter is demonstrated by Figure 6 . The figure shows pitch angles corresponding to fixed $K$ for electrons at $\mathrm{Y}=0, \mathrm{Z}=0$ and $\mathrm{X}$ from -8 to 8 for $D$ st input values from 20 to $-100 \mathrm{nT}$ at $10 \mathrm{nT}$ intervals. The other four input parameters are kept at the following constant values, $B_{z}=$ $0 \mathrm{nT}, B_{y}=0 \mathrm{nT}$, and dynamic pressure $=0.5 \mathrm{nP}$.

[34] Several features are notable. First, the sensitivity to Dst increases with radial distance. Results are little affected by $D s t$ in the inner magnetosphere where the Earth's dipole field dominates. Changes of Dst affect results more for small $K$ than for large $K$. This feature is understood because changes in Dst modify the magnetic field more near the equator than at high latitude. Particles with small $K$ remain near the equator and are most likely to be affected. Of particular significance are differences between the day and night side values. The pitch angles corresponding to fixed $K$ vary more with $D s t$ on the night side than on the dayside. The pitch angles on the nightside at $\mathrm{X}=-7$ and $K=100 \mathrm{G}^{1 / 2} \mathrm{~km}$ range from $20^{\circ}$ when $D s t=-100$ to $70^{\circ}$ when $D s t=20$. However, Polar spends much of its time off the equator measuring only particles with large $K$ values and the bulk of the analysis in this study relies on particles with $K>1000 \mathrm{G}^{1 / 2} \mathrm{~km}$. At these $K$ values the maximum variation of pitch angles calculated for models with different Dst input values is $\sim 20^{\circ}$.

[35] As mentioned previously, the pitch angles corresponding to fixed $K$ vary more as a function of $D s t$ than any other input parameters. For example, the maximum variation of pitch angles calculated for $K>1000 \mathrm{G}^{1 / 2} \mathrm{~km}$ using models with solar wind $B z$ inputs from -10 to $10 \mathrm{nT}$ is $\sim 8^{\circ}$. The maximum pitch angle variation calculated for models with $B y$ inputs from -10 to $10 \mathrm{nT}$ is $<\sim 5^{\circ}$, while models with dynamic pressure from 0 to 10 produce a maximum pitch angle variation of $\sim 10^{\circ}$. In all cases, the

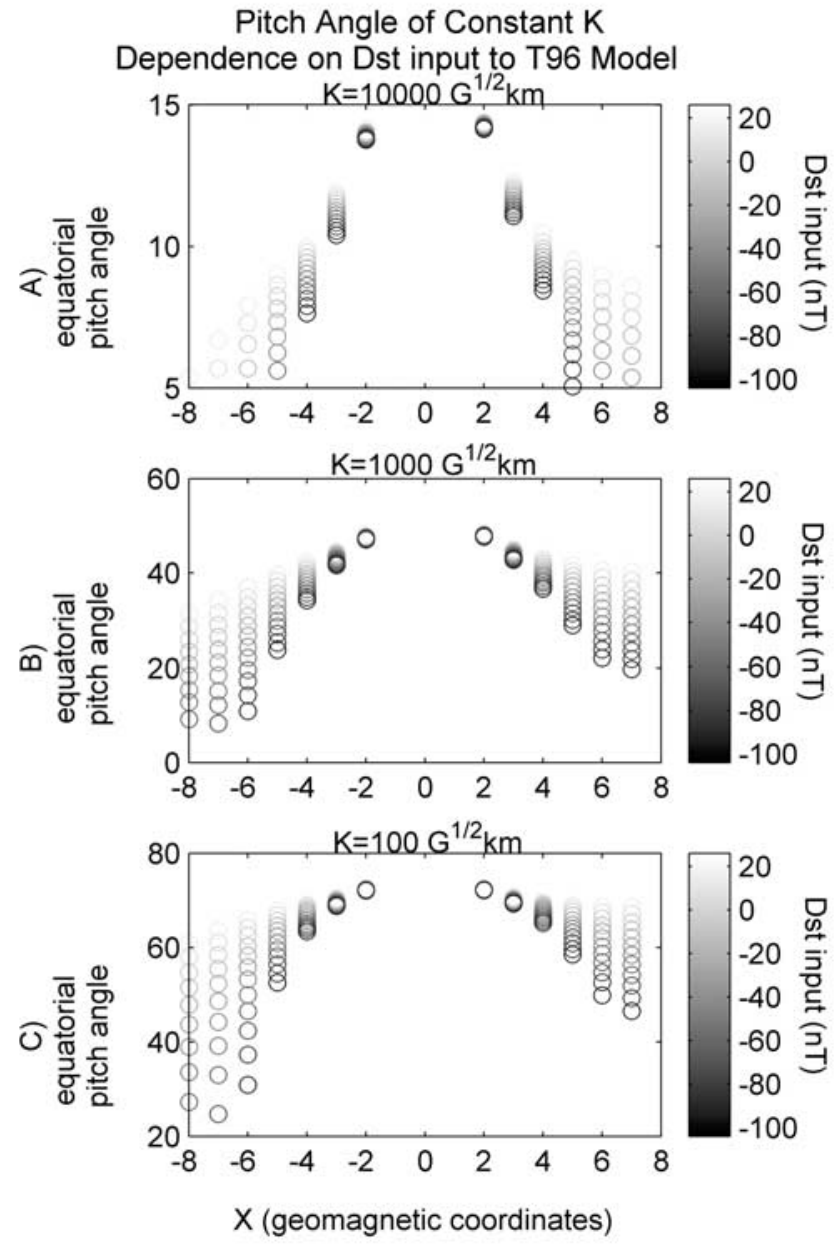

Figure 6. Plot demonstrating how the pitch angle of electrons with constant $K$ varies as the T96 magnetic field model is modified by changing only the Dst input to the model leaving other parameters fixed. The equatorial pitch angle of electrons with $K=$ (a) 10000 , (b) 1000 , and (c) $100 \mathrm{G}^{1 / 2} \mathrm{~km}$ and radial distance $X$ from -8 to 8 in geomagnetic coordinates. The pitch angle points are color coded by the value of Dst used as input to the model. Black points mark pitch angles calculated using $D s t=-100 \mathrm{nT}$ as input, and light shaded points mark pitch angles calculated using $D s t=20 \mathrm{nT}$ as input.

maximum pitch angle variation occurs at large radial distance $(r \sim 8)$ on the night side and little variation occurs in the inner magnetosphere $(r<\sim 4)$ where the Earth's dipole field dominates.

[36] Noting that Dst is the parameter that most influences the phase space density calculation, we develop the following procedure for generating a range of phase space density values that will serve as error estimates and indicate the sensitivity of our results to field model variations. To analyze each acceleration event we calculate pitch angles corresponding to constant $K$ and phase space density using the T96 field model for the measured solar wind parameters and three additional different values of Dst; the Dst measured during the event, $D s t_{\text {measured }}+40 \mathrm{nT}$, and $D s t_{\text {measured }}-40 \mathrm{nT}$. We chose to use the measured 
A)

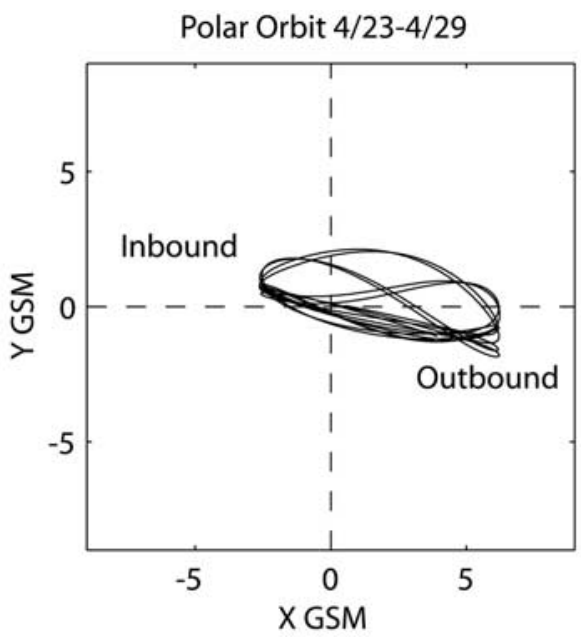

B)
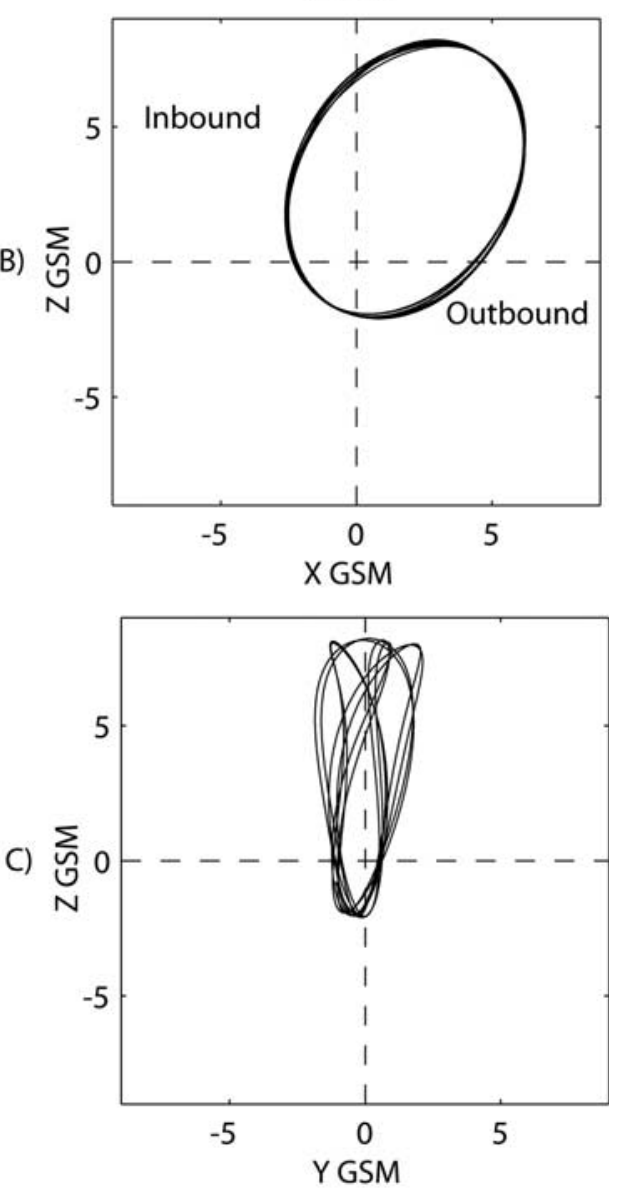

Figure 7. Polar orbit in GSM coordinates during the 23 April 1998 electron acceleration event with (a) the X-Y plane, (b) the X-Z plane, and (c) the Y-Z plane.

$D s t \pm 40 \mathrm{nT}$ because it covers $60 \%$ of the range of allowable input values and provides models from more to less geomagnetically active than the conditions encountered. An additional estimate of phase space density is obtained by selecting the value of dynamic pressure between 0.5 and $10 \mathrm{nPa}$ and the value of Dst between -100 and $20 \mathrm{nT}$ that minimizes the difference between the magnetic field measured locally at Polar and the model predicted magnetic field. The result of this procedure, which will be demon- strated in the following section, is to provide a range of phase space density values that correspond to field structures plausibly related to the probable field provided by the empirically supported T96 model.

\section{Results}

[37] The methods described above provide a set of phase space density versus $L^{*}$ profiles for acceleration events sampled over a range of local times between January 1997 and January 1999. The results that we present in this section appear to show that the evolution of phase space density depends on local time. Events relying on data obtained from the nightside magnetosphere have outward phase space density gradients consistent with radial diffusion and external acceleration. However, events measured on the dayside magnetosphere have phase space density profiles with peaks inside of geostationary orbit, consistent with only internal acceleration. Similar day/night asymmetries were observed in the study by Selesnick and Blake [2000]. In neither case are such asymmetries likely to arise from physically realistic effects as we discuss below. We focus on two example acceleration events that contrast the day and nightside results. We discuss how imperfect field models cause phase space density errors that may explain the observed local time differences. Finally, we identify the observations that should be least affected by phase space density errors and discuss implications for acceleration models.

\subsection{Local Time Dependence of Phase Space Density}

\subsubsection{Dayside Phase Space Density Profiles}

[38] For the storm whose main phase began on 2300 UT 23 April 1998 we have obtained phase space density profiles of electrons with $K$ values from 500 to $5000 \mathrm{G}^{1 / 2}$ $\mathrm{km}$ and $\mu$ values from 200 to $2000 \mathrm{MeV} / \mathrm{G}$. In this event, the data are useful principally for the dayside because of the tilted orbit. The orbit is plotted in GSM coordinates from 23 to 29 April 1998 in Figure 7. Figure 7a shows the X-Y GSM plane. The inbound portion of the orbit samples the nightside radiation belts at local times from 2300 to 2400 . Figures $7 \mathrm{~b}$ and $7 \mathrm{c}$ show the X-Z and Y-Z GSM plane. The nightside portion of the orbit rises sharply, moving above the mirror point of electrons with $\mathrm{K}<5000 \mathrm{G}^{1 / 2} \mathrm{~km}$ and limiting the measured data to $L^{*}<\sim 4.5$. The outbound portion of the orbit samples the dayside radiation belts at local times from 1100 to 1300 and reaches $L^{*}$ values up to $\sim 8$. Thus our analysis for this event focuses on the day side because extended radial coverage is necessary to identify the shape of the radial gradient.

[39] The range of $K$ values chosen for study is not arbitrary. The values measured are a function of the Polar orbit as well as the pitch angle and energy resolution of the HIST instrument. We limit our study to $K$ values less than $5000 \mathrm{G}^{1 / 2} \mathrm{~km}$ because of the instrument pitch angle resolution. HIST has $22^{\circ}$ pitch angle bins and does not resolve the flux of electrons with equatorial pitch angles smaller than this bin width. Therefore, the measured phase space density of electrons with $K>5000 \mathrm{G}^{1 / 2} \mathrm{~km}$ values is not reliable.

[40] Figure 8 shows phase space density of electrons with $K=2500 \mathrm{G}^{1 / 2} \mathrm{~km}$ and $\mu=1000 \mathrm{MeV} / \mathrm{G}$ versus $L^{*}$ (from 3 to 8 ) along with solar wind and Dst data throughout the 

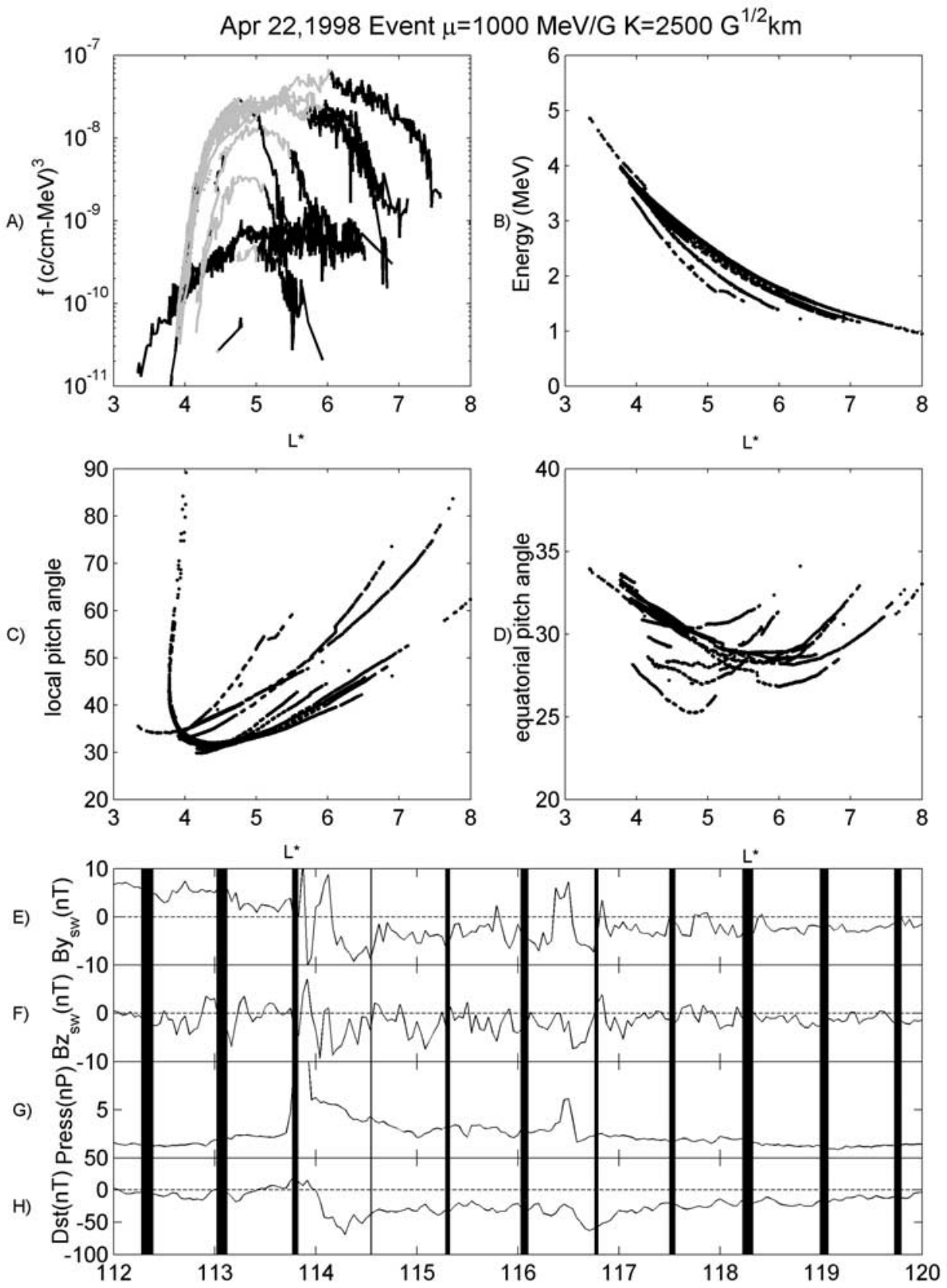

Day of year 1998

Figure 8. Example of an acceleration event that relies predominantly on data taken from the dayside portion of the Polar orbit and shows increasing phase space density peaks at intermediate $L^{*}$ indicative of internal acceleration. (a) Phase space density of electrons in units of $(\mathrm{c} / \mathrm{cm}-\mathrm{MeV})^{3}$ where $\mathrm{c}$ is the speed of light for $\mu=1000 \mathrm{MeV} / \mathrm{G}$ and $K=2500 \mathrm{G}^{1 / 2} \mathrm{~km}$ from 23 to 29 April. The data are plotted versus $L^{*}$ and are color coded in time with each color corresponding to a different orbit. (b) The energy $(\mathrm{MeV})$ of electrons used in the calculation of phase space density. (c) The local pitch angle (degrees) of electrons used in the calculation and (d) the equatorial pitch angle (degrees) of the electrons. (e) The $B_{y}$ (nT) component and (f) the $B_{z}(\mathrm{nT})$ component of the solar wind in GSM coordinates. (g) The dynamic pressure (nP) and (h) Dst (nT). Vertical bars mark the times during the Polar orbit that the radiation belts were encountered. These bars are color coded to match the data plotted in Figures 8a-8d. See color version of this figure at back of this issue. 

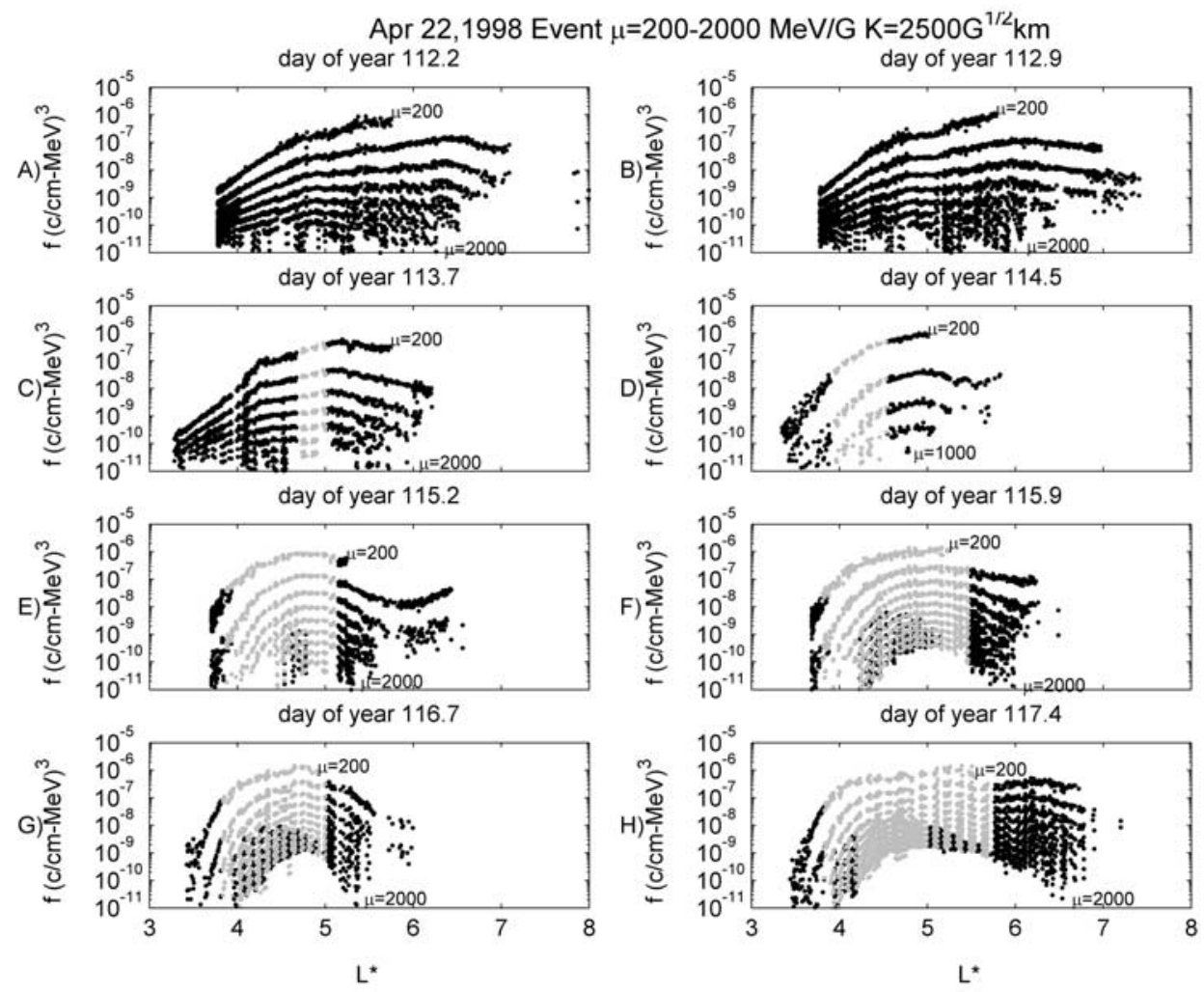

Figure 9. Phase space density $(\mathrm{c} / \mathrm{cm}-\mathrm{MeV})^{3}$ of electrons with $K=2500 \mathrm{G}^{1 / 2} \mathrm{~km}$ and $\mu=200$ $2000 \mathrm{MeV}$. (a)-(h) Data from orbits passing through the radiation belts on days 112 to 117 .

23 April 1998 event. Figure 8a shows electron phase space density versus $L^{*}$ with each trace color coded in time. Prior to the storm the phase space density has a positive slope that peaks at large $L^{*}$. Just after the main phase on day 114 the phase space density decreases below the detection threshold of the instrument. As the phase space density increases during the recovery phase of the storm the profiles change. On day 115 the phase space density peaks at $L^{*}=5$. As the storm continues, the maximum phase space density increases and extends to higher $L^{*}$ values. These increasing phase space density peaks observed on the day side are consistent with internal acceleration of electrons and not with acceleration by radial diffusion from a source outside of geostationary.

[41] Figure 9 shows electron phase space density versus $L^{*}$ for a range of $\mu$ values. Figures $9 \mathrm{a}-9 \mathrm{~h}$ show phase space density of electrons with $\mu$ from 200 to $2000 \mathrm{MeV} / \mathrm{G}$ in increments of $200 \mathrm{MeV} / \mathrm{G}$ and $K=2500 \mathrm{G}^{1 / 2} \mathrm{~km}$ during each pass through the radiation belts. An electron with $K=$ $2500 \mathrm{G}^{1 / 2} \mathrm{~km}$ and $\mu=200 \mathrm{MeV} / \mathrm{G}$ at $L^{*}=6$ has an energy of $\sim 0.8 \mathrm{MeV}$. An electron with $K=2500 \mathrm{G}^{1 / 2} \mathrm{~km}$ and $\mu=$ $2000 \mathrm{MeV} / \mathrm{G}$ at $L^{*}=6$ has an energy of $\sim 2.5 \mathrm{MeV}$. During the early recovery phase (days $115-116$ ) the phase space density at all $\mu>200 \mathrm{MeV} / \mathrm{G}$ increases forming peaks at low $L^{*} \sim 5$. The phase space density with $\mu=200 \mathrm{MeV} / \mathrm{G}$ is restricted to $L^{*}<5$ making the determination of a peak difficult. By day $117 \mu$-dependent differences in the phase space density profiles such as were described by Brautigam and Albert [2000] appear. On day 117 the phase space density peak for electrons with $\mu=400 \mathrm{MeV} / \mathrm{G}$ has moved outside $L^{*}>6.5$, while phase space density peaks at $L^{*}=5$ are still evident at higher $\mu$ values.

[42] Figure 10 shows electron phase space density with $\mu=1000 \mathrm{MeV} / \mathrm{G}$ over a range of $K$ values from 500 to 5000 $\mathrm{G}^{1 / 2} \mathrm{~km}$ in increments of $500 \mathrm{G}^{1 / 2} \mathrm{~km}$ for the same event. An electron with $K=500 \mathrm{G}^{1 / 2} \mathrm{~km}$ at $L^{*}=6$ has an equatorial pitch angle of $\sim 55^{\circ}$ and an electron with $K=$ $5000 \mathrm{G}^{1 / 2} \mathrm{~km}$ at $L^{*}=6$ has an equatorial pitch angle of $\sim 20^{\circ}$. During the recovery phase (days $115-118$ ) electrons with $K>1000 \mathrm{G}^{1 / 2} \mathrm{~km}$ show phase space density increases forming peaks at $L^{*} \sim 5$ consistent with internal acceleration. For electrons with $K<1000 \mathrm{G}^{1 / 2} \mathrm{~km}$ the range of $L^{*}$ measured is restricted to $L^{*}<5$ because the Polar orbit passes above the mirror point of these electrons. No phase space density peaks are observed for $K<1000 \mathrm{G}^{1 / 2} \mathrm{~km}$ inside $L^{*}=5$ but peaks at larger $L^{*}$ cannot be ruled out.

\subsubsection{Nightside Phase Space Density Profiles}

[43] Next, we analyze phase space density profiles determined from a storm whose main phase began on 24 September 1998. This event provides phase space density profiles in the nightside magnetosphere. Figure 11 shows the orbit from 23 to 30 September in GSM coordinates. The inbound portion of the orbit samples the dayside radiation belts at local times between $\sim 1100$ and $\sim 1300$ and the outbound portion of the orbit samples the nightside radiation belts at local times between $\sim 0000$ and $\sim 0200$. This event gives the most extensive radial coverage in the local time region near midnight. Figure 12 shows phase space density of electrons with $\mu=1000 \mathrm{MeV} / \mathrm{G}$ and $K=$ $2500 \mathrm{G}^{1 / 2} \mathrm{~km}$ along with the solar wind data and the Dst 

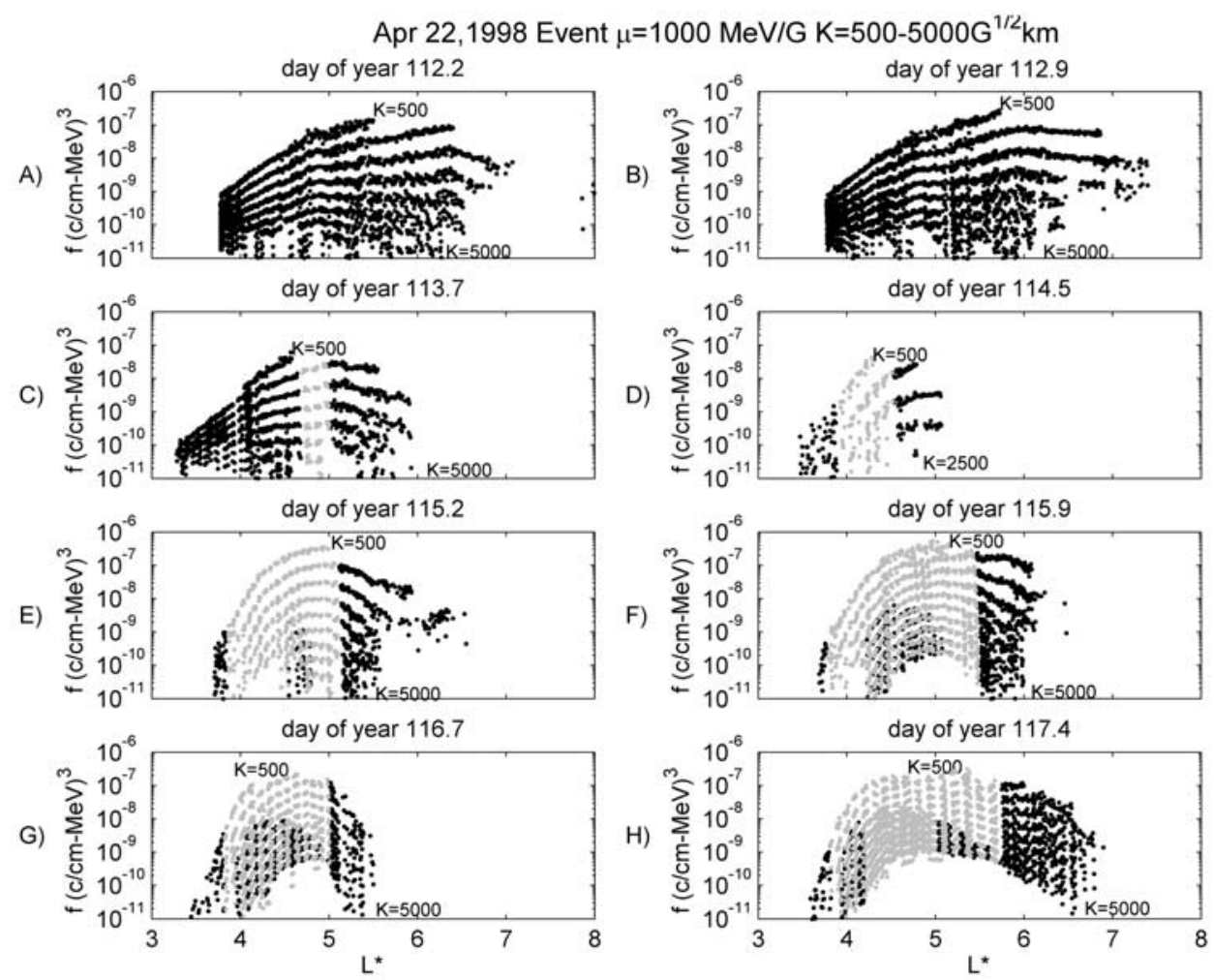

Figure 10. Phase space density $(\mathrm{c} / \mathrm{cm}-\mathrm{MeV})^{3}$ of electrons with $\mu=1000 \mathrm{MeV}$ and $K=500-5000 \mathrm{G}^{1 / 2} \mathrm{~km}$. (a)-(h) Data from orbits passing through the radiation belts on days 112 to 117.

index. Figure 12a shows the phase space density versus $L^{*}$ for each orbit color coded by time. In this event the phase space density increases with time with a maximum always at large $L^{*}$. During the period of acceleration from day 270 to 273 we do not observe any increasing phase space density peaks inside of $L^{*}=7$. Figure 13 shows phase space density of electrons with $K=2500 \mathrm{G}^{1 / 2} \mathrm{~km}$ and $\mu$ ranging from 200 to $2000 \mathrm{MeV} / \mathrm{G}$. Figure 14 shows phase space density of electrons with $\mu=1000 \mathrm{MeV} / \mathrm{G}$ and $K=$ $500-5000 \mathrm{G}^{1 / 2} \mathrm{~km}$. The phase space density versus $L^{*}$ profiles in all three figures predominantly show outward gradients for $L^{*}$ between 4 and 8 consistent with acceleration by radial diffusion form a source at large $L^{*}$. An exception is seen on day 271.3 where at the highest $K$ values $\left(3000-5000 \mathrm{G}^{1 / 2} \mathrm{~km}\right)$ a modest negative gradient between $L^{*} \sim 6$ and $L^{*} \sim 7.5$ is observed.

[44] One might consider the possibility that the phase space density profiles differ for the two storms simply because different processes accelerate electrons in each event. However, a survey of 2 years of data shows that the inferred structure of phase space density versus $L^{*}$ varies systematically with local time. Figure 15 shows 12 acceleration events occurring within the 2 years as well as the local time of each orbit. For each event we only plot data from the day or nightside portion of the orbit that gives extended radial coverage. Events plotted in Figures $15 \mathrm{a}-15 \mathrm{~h}$ all show evidence of an increasing phase space density peak at $L^{*} \sim 5$ indicative of internal acceleration. The local time plot shows that for these events the data were obtained predominantly from $0500<$ MLT $<1900$. The events plotted in Figures 15i-151 are more erratic but show evidence of phase space density maximums at $L^{*} \sim 8$ as would be expected for radial diffusion from an external source. These events contain data obtained from MLT $>2000$ and MLT $<0300$. It is statistically improbable that radial diffusion events occurred only while Polar traversed the nightside magnetosphere and internal acceleration events occurred while Polar traversed the dayside magnetosphere. Therefore, some explanation for the unusual systematic day-night asymmetry must be found.

\subsection{Understanding Local Time Differences}

[45] The observed local time differences of phase space density admit of different interpretations. One interpretation is that the dayside and nightside phase space density profiles differ because the structure of the dayside magnetosphere produces unusual particle drift orbits known as Shabansky orbits [Shabansky, 1971] that modify the phase space density gradients. Another interpretation is that the phase space density profiles are produced by acceleration and loss processes that vary with local time. The last and most probable interpretation is that the local time differences result from systematic errors in phase space density calculations.

[46] We can reject the hypothesis that the dayside phase space density gradients are modified by particles following Shabansky orbits because these unusual orbits are not expected in the magnetospheric region examined in this study. Shabansky orbits are trajectories that occur when off equatorial particles drift from nightside field lines that have a single equatorial minimum onto outer dayside field lines 


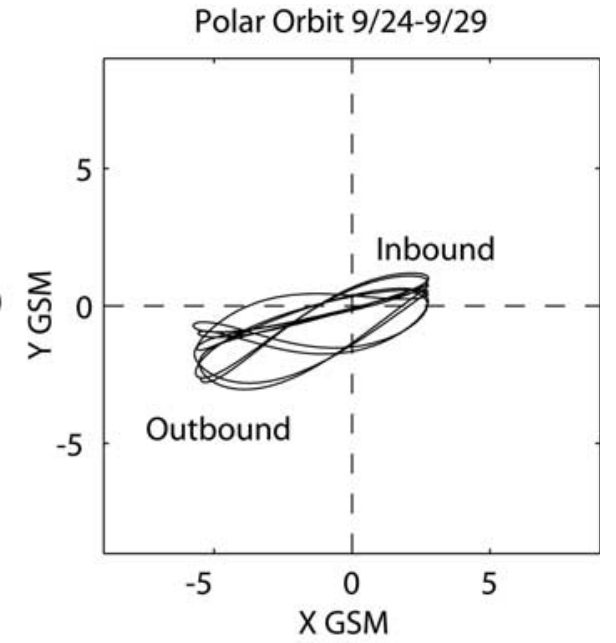

B)
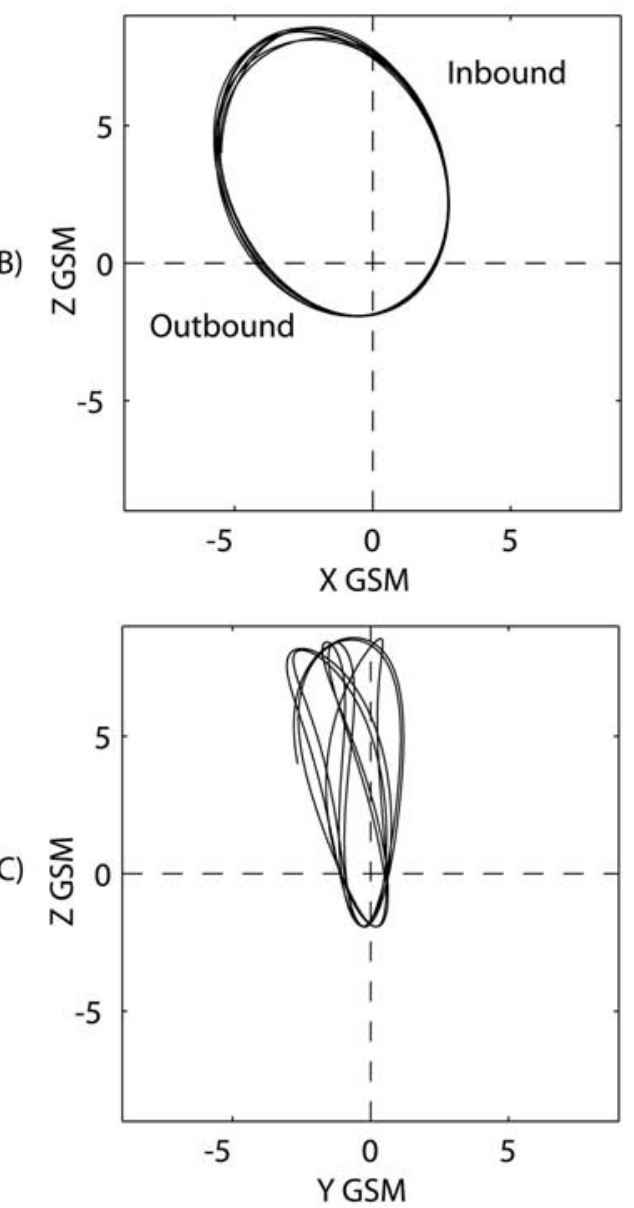

Figure 11. Polar orbit during the 24 September electron acceleration event: the orbit in (a) the X-Y plane, (b) the $\mathrm{X}-\mathrm{Z}$ plane, and (c) the Y-Z plane in GSM coordinates.

field lines that have multiple magnetic minima at the equator and the cusp region. On the nightside field lines, the mirror force causes off equatorial particles to bounce about the equatorial minimum. When the particles drift to dayside field lines with multiple minima, the mirror force reverses direction and pushes the particles off the equator to bounce about the field minimum near the cusp region. In some cases the motion may violate the second adiabatic invariant. To determine whether Shabansky orbits affect the phase space density data shown in this study we traced magnetic field lines in the T96 field model through the position of Polar during the dayside 23 April event shown in Figure 8. According to the T96 field model, Polar was not located on field lines with multiple minima during the time this data was taken. Thus Shabansky orbits can not explain the differences in the dayside and nightside phase space density gradients.

[47] Our arguments for rejecting the hypothesis that local time differences arise from a combination of radial diffusion from an external source and localized losses relies heavily on a comparison of particle drift periods with source and loss timescales. The proposed mechanism requires that electrons be accelerated and transported from a source region in the nightside magnetotail. As the electrons drift eastward toward the dayside, preferential loss occurs at large $L^{*}$ producing a peak at low $L^{*}$. Possible loss mechanisms include precipitation into the atmosphere or loss through the magnetopause boundary to the solar wind. The lost electrons are once again replenished on the nightside so that the outward radial gradient is reestablished. In order to maintain the day/night asymmetry over many orbits, as observed, the electron loss and replenishment must occur on timescales shorter than the electron drift period. At $L^{*}=7$ electrons with energy $>0.7 \mathrm{MeV}$ have full drift periods less than $30 \mathrm{~min}$. Lorentzen et al. [2001] estimate that without a new source of electrons, microburst precipitation could deplete the entire outer radiation belt during the course of a single storm. The study suggests that noticeable losses could occur on timescales of the order of $30 \mathrm{~min}$. Current estimates of radial diffusion timescales, on the other hand, range from several days to several hours [e.g., Elkington et al., 2003] suggesting that this interpretation is unlikely. However, estimates of radial diffusion timescales depend on wave power and the chosen theoretical form of the diffusion coefficient. More detailed measurements of wave power as a function of the azimuthal wave structure, frequency, magnetic latitude, and geomagnetic activity combined with more accurate forms of the diffusion coefficient are required to precisely define the radial diffusion timescales.

[48] We can readily argue that the final hypothesis, i.e., that the observed local time differences arise from erroneous phase space density calculations, is reasonable. As explained previously, there are two sources of errors in the calculation of phase space density; poor data fits and imperfect magnetic field models. The data fits are not a problem, as we show by examining changes that would be consistent with errors determined from the boot strap method. Figure 16 shows phase space density measured on the day side for 1 day (116) during the April 1998 storm along with the $95 \%$ confidence ranges introduced in fitting the pitch angle and energy distributions. These error ranges are not significant compared with the magnitude of phase space density changes. Figure 17 shows an example from a nightside pass on day 272 obtained during the September 1998 storm. Errors from fitting the pitch angle distributions or the energy spectrum could not change the gradient enough to produce a peak at intermediate $L^{*}$. 

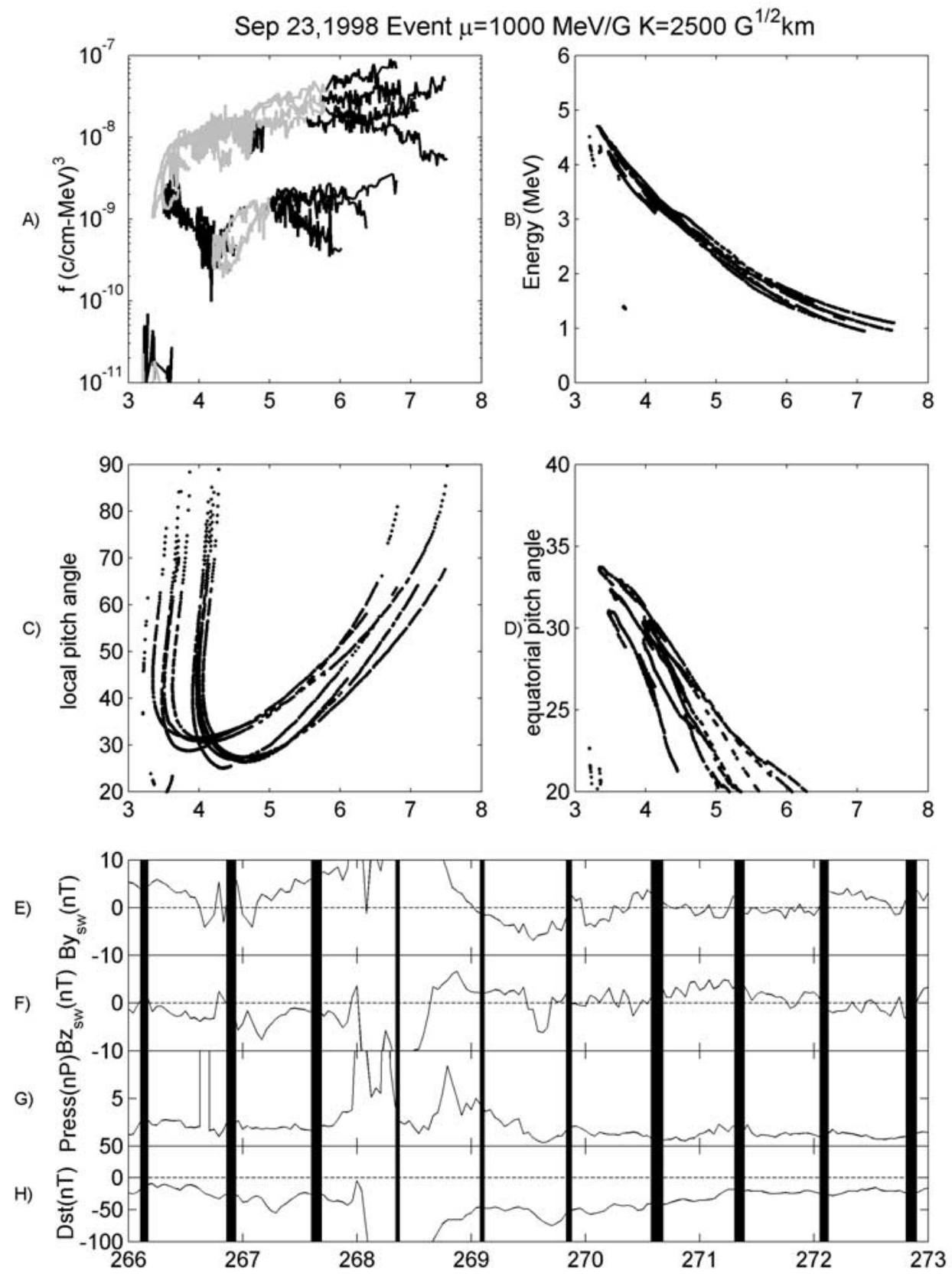

Day of year 1998

Figure 12. Example of an acceleration event that relies predominantly on data taken from the nightside portion of the Polar orbit and shows phase space density gradients indicative of external acceleration. The format is the same as for Figure 8. See color version of this figure at back of this issue.

[49] Imperfect field models can, on the other hand, modify gradients of phase space density significantly. As discussed previously, imperfect magnetic field models change the calculation of $K$. The erroneous $K$ values in turn change the phase space density versus $L^{*}$ profile and may create artificial phase space density peaks. Section 2.3 introduced methods for analyzing phase space density errors introduced by incorrect estimates of $K$. We apply those methods here.

[50] Figure 18 illustrates how phase space densities are affected by inaccurate calculations of $K$ for the two electron acceleration event discussed previously. The figure shows phase space density calculated using the four different outputs of the T96 field model corresponding to the measured solar wind and the measured Dst, Dst $t_{\text {measured }}+$ $40 \mathrm{nT}$, and $D s t_{\text {measured }}-40 \mathrm{nT}$, and the parameters for which the model matches the locally measured $B$ (see section 2.3).

[51] Similarities and differences exist among the phase space density profiles calculated using the various models. The most striking similarity is that on the dayside (Column A) all four traces show phase space density 

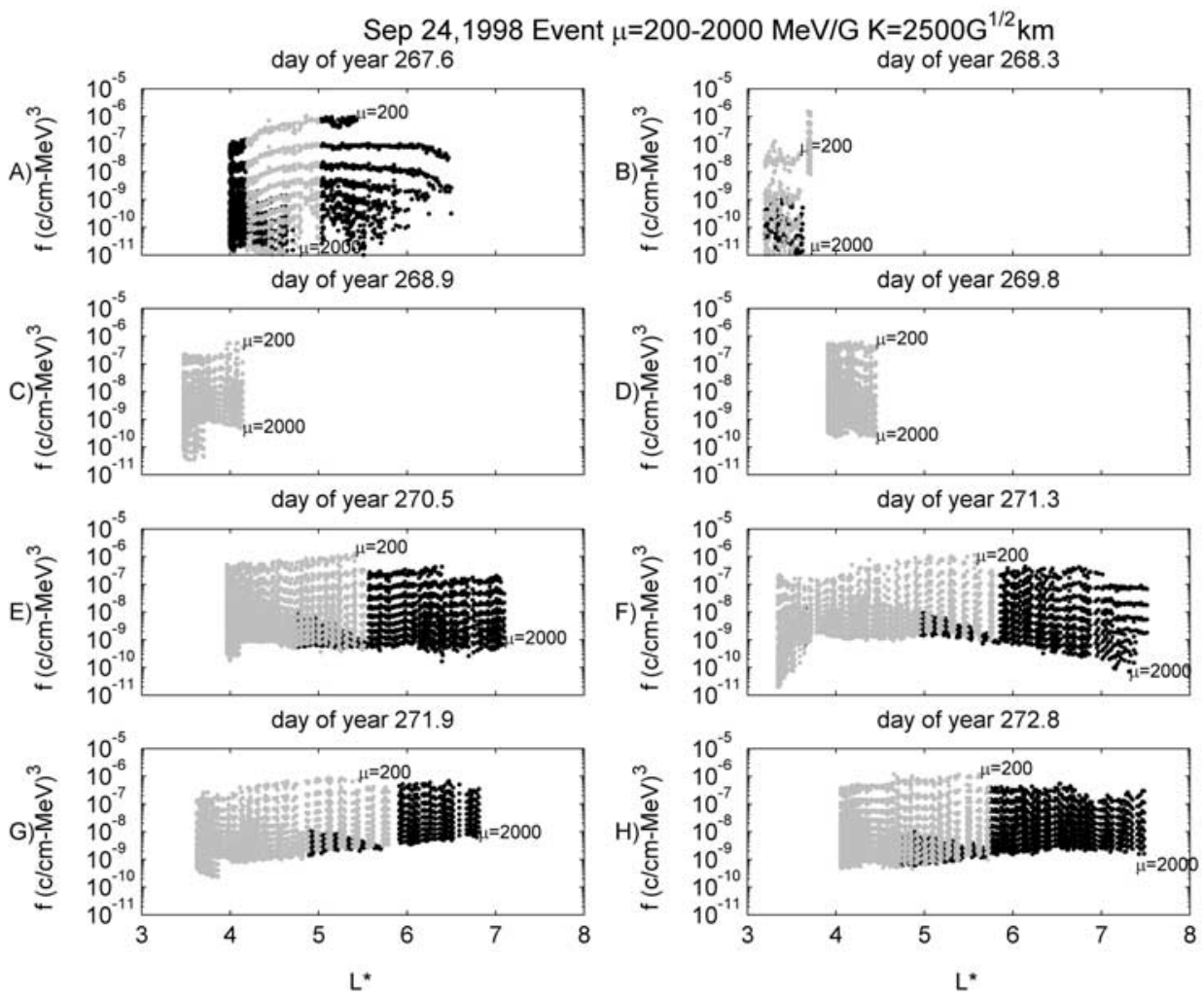

Figure 13. Phase space density $(\mathrm{c} / \mathrm{cm}-\mathrm{MeV})^{3}$ of electrons with $K=2500 \mathrm{G}^{1 / 2} \mathrm{~km}$ and $\mu=200$ $2000 \mathrm{MeV}$. (a)-(h) Data from orbits passing through the radiation belts on days 262 to 267 .

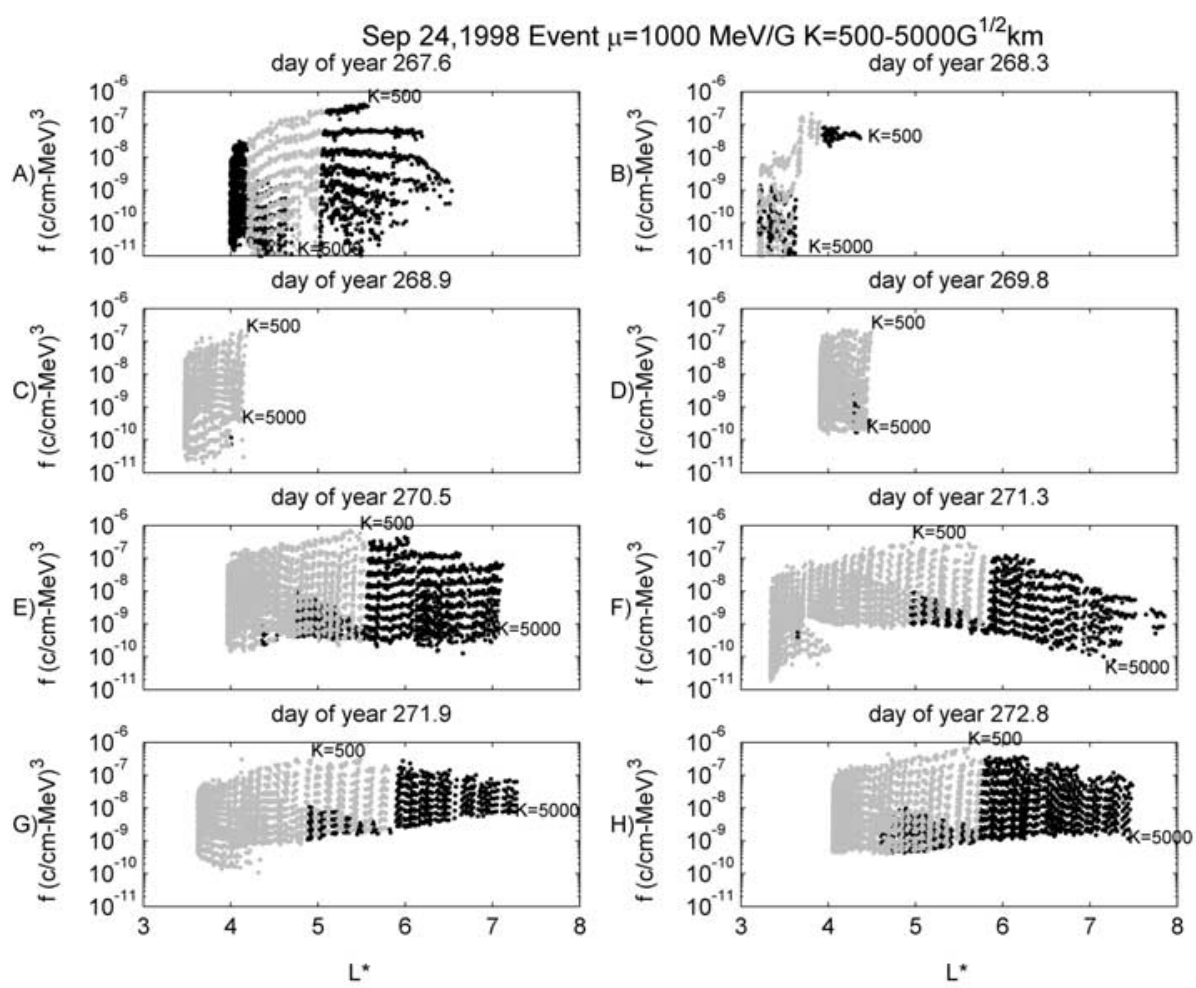

Figure 14. Phase space density $(\mathrm{c} / \mathrm{cm}-\mathrm{MeV})^{3}$ of electrons with $\mu=1000 \mathrm{MeV}$ and $K=500$ $5000 \mathrm{G}^{1 / 2} \mathrm{~km}$ and. (a)-(h) Data from orbits passing through the radiation belts on days 262 to 267 . 


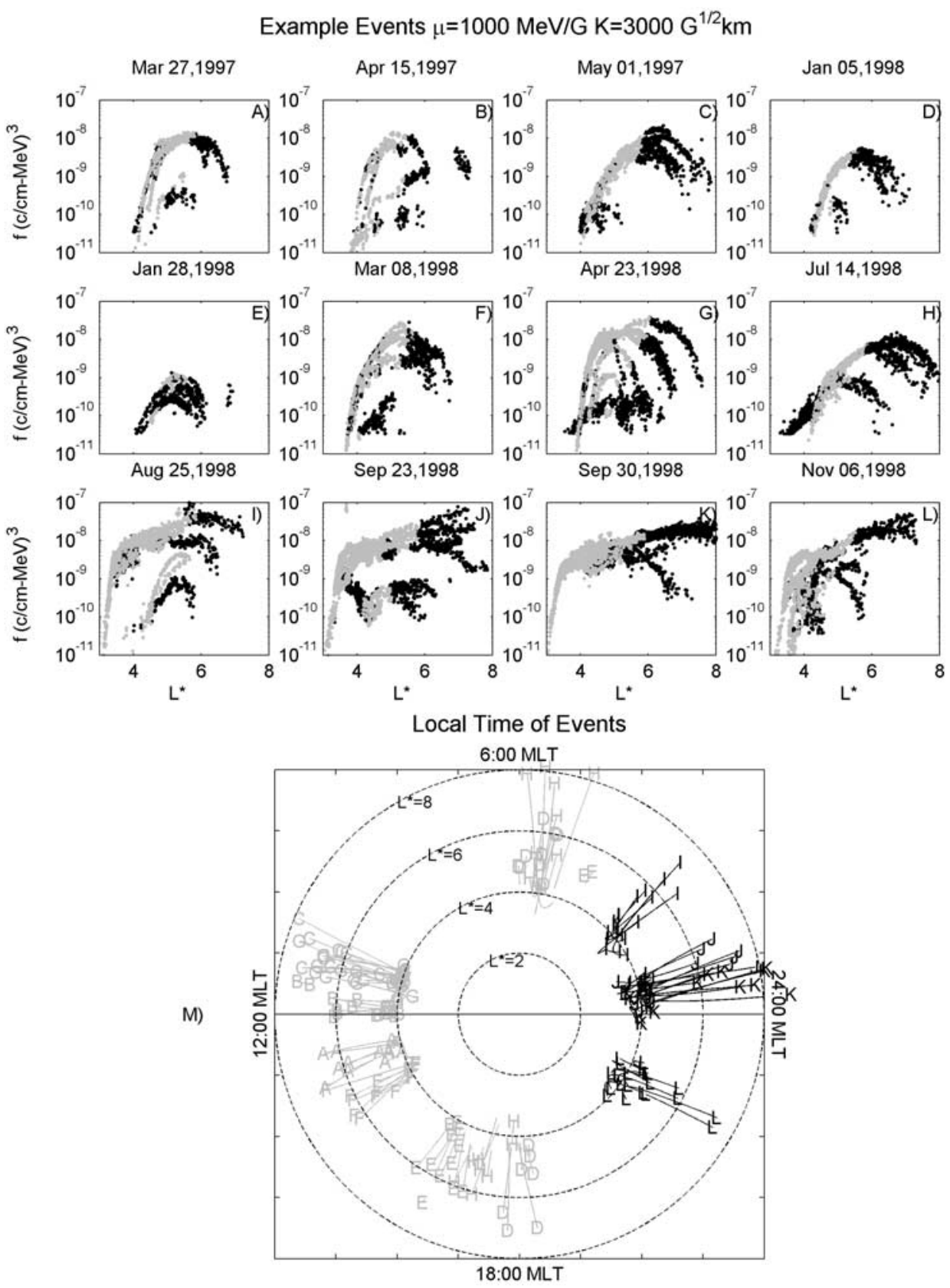

Figure 15. Demonstration of the local time dependence of phase space density versus $L^{*}$ profiles. (a)-(l) Phase space density (c/cm-MeV) 3 versus $L^{*}$ for 12 events from January 1997 to January 1999. (m) The local time of each event. Each trace in Figure $15 \mathrm{~m}$ is labeled with a letter corresponding to the event plotted in Figures 15a-151. See color version of this figure at back of this issue.

peaks at $L^{*} \sim 5$ after day 115 during the recovery phase of the storm. The fact that the peak remains regardless of the field model suggests this is a robust result. However, the profiles show differences that deserve comment. In the inner magnetosphere at $L^{*}<\sim 5$ the phase space density calculated using $D s t_{\text {measured }}+40 \mathrm{nT}$ (plus symbols) as input is higher than the phase space density calculated using $D s t_{\text {measured }}-40 \mathrm{nT}$ (dashed trace) as input to the model. In the outer magnetosphere where $L^{*}>5$ the opposite relation is true. At these larger $L^{*}$ values the phase space density calculated using $D s t_{\text {measured }}{ }^{+}$ $40 \mathrm{nT}$ (plus symbols) as input is lower than the phase space density calculated using $D s t_{\text {measured }}-40 \mathrm{nT}$ (dashed trace) as input to the model.

[52] The different results are understood by comparing the model magnetic field to the measured magnetic field at the position of Polar predicted for the two inputs. Figure 19a shows phase space density for 26 April 1998 (day 116) obtained using the different outputs of the T96 model. Figure $19 \mathrm{~b}$ shows $B_{\text {meas }} / B_{\text {model }}$ where $B_{\text {meas }}$ is the magnetic field measured locally by the MFE instrument and $B_{\text {model }}$ is the magnetic field predicted by the T96 models. In the inner magnetosphere $L^{*}<\sim 5.2$ the T96 model obtained using $D s t+40 \mathrm{nT}$ (plus symbols) as input overestimates the local 


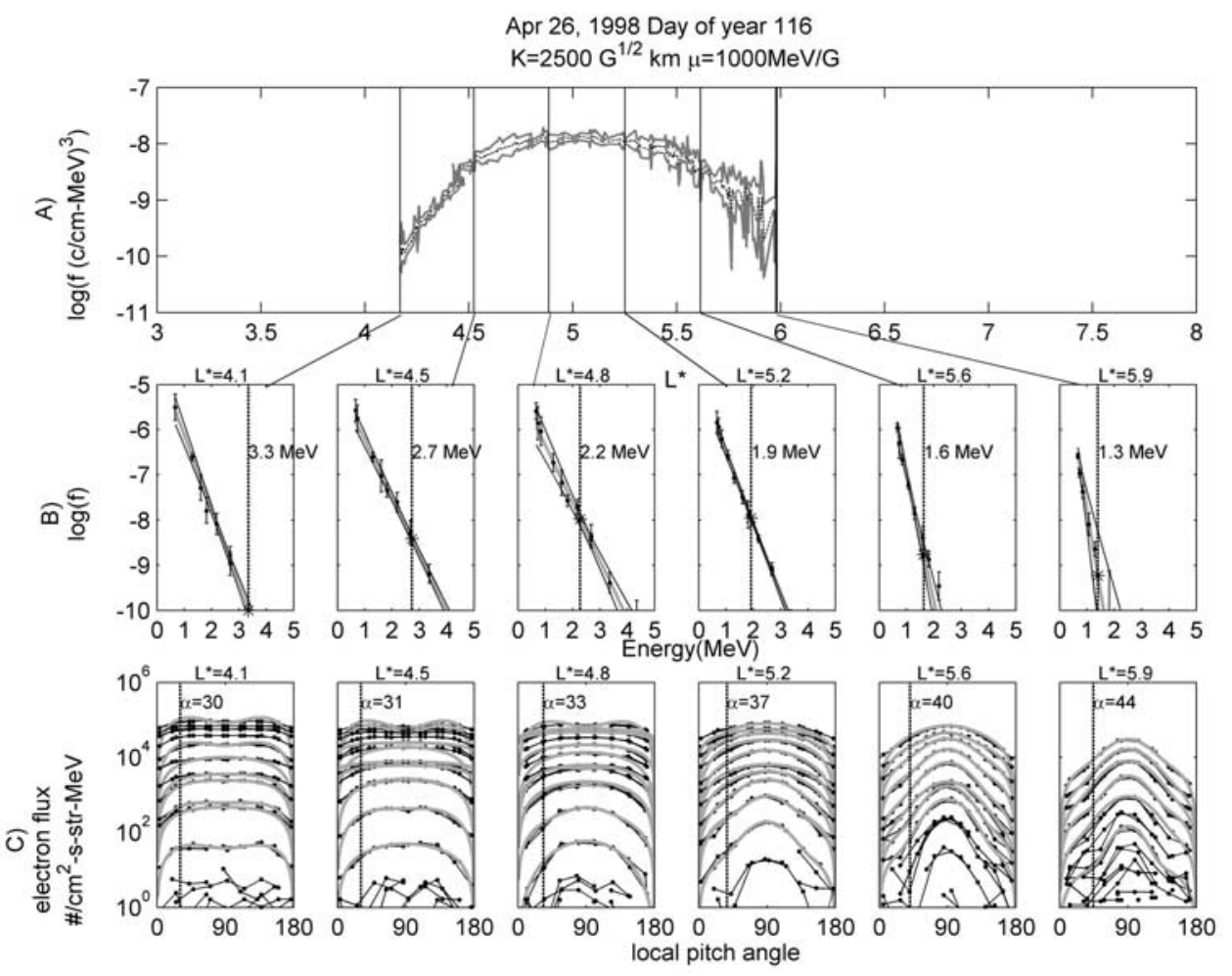

Figure 16. Phase space density errors introduced from data fits. (a) Phase space density (c/cm-MeV) ${ }^{3}$ with error bars during a dayside pass on day 116 . The black trace shows phase space density. The shaded trace shows the bootstrap errors associated with fitting the energy distributions. (b) The log of phase space density versus energy at various $L^{*}$ distances. Measured phase space density is plotted as black points with error bars showing the bootstrap error from the pitch angle distributions. The shaded line represents a fit to the data, and the dashed line shows the phase space density at the energy of constant $\mu$. (c) Differential flux versus pitch angle at 14 different energies. The black points show the measured data, and the shaded lines show the data fits. The dashed line marks the pitch angle corresponding to constant $K$.

magnetic field. The phase space density calculated using this model is also high (plus symbols). The T96 model obtained using Dst - $40 \mathrm{nT}$ (dashed trace) underestimates the magnetic field and the calculated phase space density is low. For $L^{*}>\sim 5$ the model predictions are reversed but the same relation between the phase space density and the magnetic field still holds. When the model overestimates the local magnetic field, the calculated phase space density is high and conversely when the model underestimates the local magnetic field, the calculated phase space density low. The relationship is understood by using a very crude approximation for the second invariant: $K=\left[B_{\text {mirror }}-\right.$ $\left.B_{\text {equator }}\right]^{1 / 2} s$. Here $B_{\text {mirror }}$ is the off equatorial mirror point magnetic field, $B_{\text {equator }}$ is the equatorial field and $s$ is the distance between the mirror points. If the local field is overestimated by the model, then $K$ and phase space density are also likely to be overestimated. This is the relationship seen in Figure 19. The estimated phase space density increases monotonically with $B_{\text {measured }} / B_{\text {model }}$.

[53] The dayside phase space density profiles are not very sensitive to errors in $K$ but the nightside profiles are highly dependent on the chosen field model. Figure 18 column B shows the phase space density from the nightside magnetosphere during the 23 September 1998 storm. The data are plotted in the same format as in the previous figure and use the same procedure for estimating phase space density errors introduced by the magnetic field model. In this case large differences exist among the four traces. The phase space density obtained using $D s t+40 \mathrm{nT}$ as input to the model shows a phase space density peak at $L^{*}=5$ on days 270 and 271 consistent with internal acceleration. In contrast, the phase space density obtained on these same days using $D s t_{\text {measured }}-40 \mathrm{nT}$ shows an outward gradient consistent with radial diffusion from an external source. The phase space density obtained using the field model that best matches the magnetic field measured locally by Polar also has an outward gradient. The discrepancy demonstrates that the nightside calculations are highly dependent on the field model. The sensitivity is explained using the same crude approximation of $K=\left[B_{\text {mirror }}-B_{\text {equator }}\right]^{1 / 2} s$. The nightside region is very sensitive to the field model because even if $B_{\text {mirror }}$ is well estimated, $B_{\text {equator }}$ varies considerably with the stretching of the tail. A more stretched field implies larger $K$ values and a higher estimate of the phase space density. Thus the estimates of phase space density at the largest $L^{*}$ values can easily be overestimated and produce spurious outward gradients. Stretching is not a problem on dayside field lines.

\section{Discussion}

[54] Using the T96 model with the measured solar wind and Dst as input, radial profiles of electron phase space 


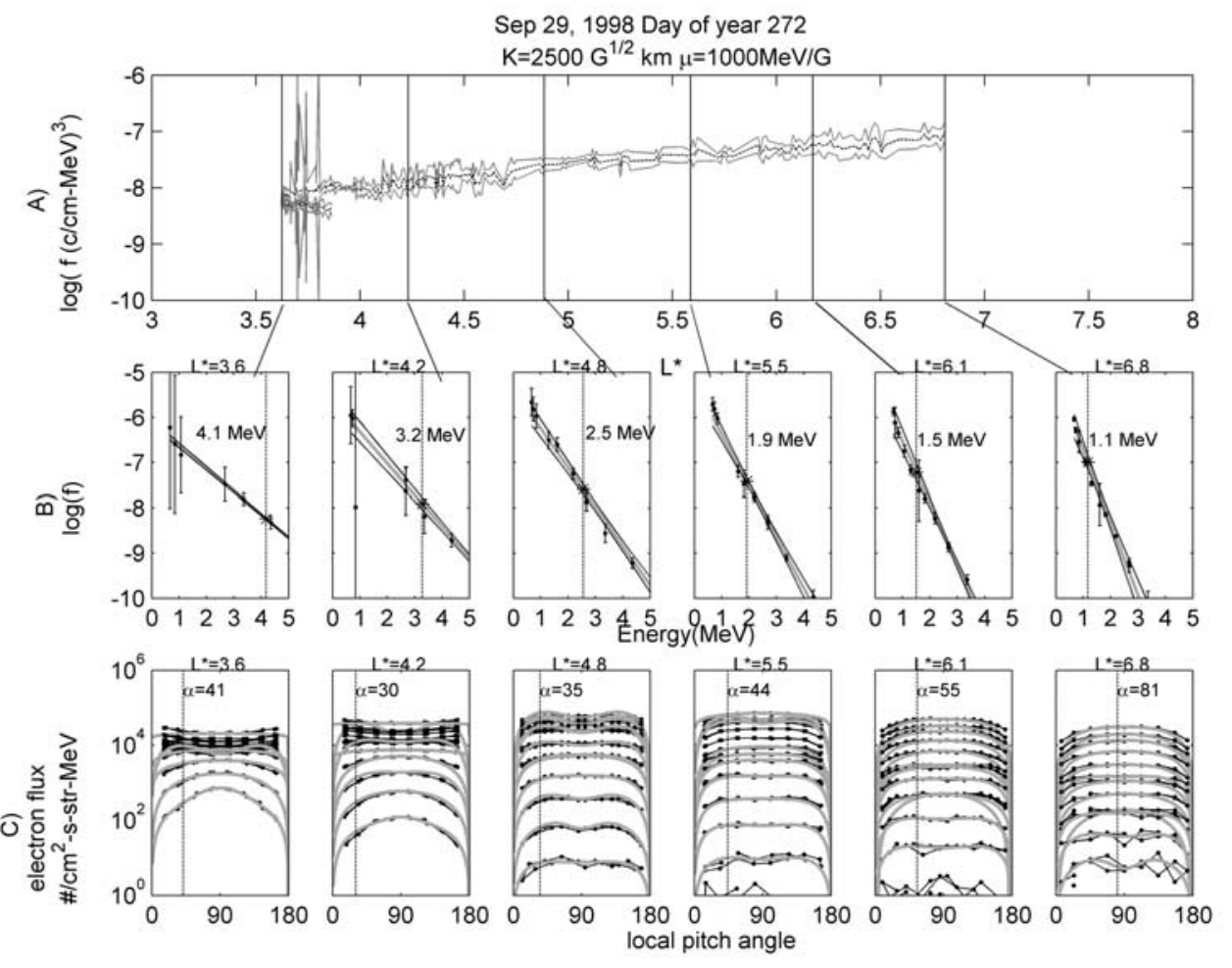

Figure 17. Phase space density errors introduced from data fits. The format of the plot is the same as Figure 16, but the data are obtained from a dayside pass on day 116.

density obtained from dayside measurements are consistent with internal acceleration mechanisms. Radial profiles of electron phase space density obtained from nightside measurements are consistent with external acceleration mechanisms. The discrepancy is explained by the sensitivity of phase space density calculations to the chosen magnetic field model rather than a physical phenomenon. Phase space densities obtained from dayside measurements are rather insensitive to changes of the field model but those from nightside measurements are highly sensitive to the field model adopted. Calculating phase space density on the nightside using a less stretched field model results in radial profiles consistent with internal acceleration while using highly stretched field models results in profiles consistent with radial diffusion. The nightside magnetic field is subject to dynamic processes that change the stretching of the tail. A static field such as T96 model does not capture these dynamic processes; therefore, the nightside phase space density calculations are not reliable. In contrast, the dayside phase space density estimates show peaks consistent with internal acceleration regardless of the field model used.

[55] It must be noted that the results of this study apply to electrons mirroring off the equator with equatorial pitch angles less than $\sim 45^{\circ}$. It is possible that the phase space density gradients of equatorial and off-equatorial electrons differ. A scenario that could explain our results is one whereby radial diffusion accelerates and transports equatorial electrons from a source at large $L^{*}$ which are then scattered to smaller pitch angle in the inner magnetosphere. However, the phase space density gradients obtained by Brautigam and Albert [2000], although not completely conclusive, imply that near equatorial electrons with $\mu>316 \mathrm{MeV} / \mathrm{G}$ are also accelerated by an internal source mechanism.

[56] Our results are also compatible with other previous investigations even those that at first glance seem conflicting. For example, Hilmer et al. [2000] found higher phase space density at $L=6.6$ than at $L=4.2$ for electrons with $K=0$, which they took as evidence for external acceleration. The event times and $K$ values used by Hilmer et al. [2000] differ from those in our study making direct detailed comparisons impossible but qualitative similarities are worth noting. Figures $15 \mathrm{a}-15 \mathrm{~h}$, which present robust dayside events, indeed show higher phase space density at $L^{*}=6.6$ than at $L^{*}=4.2$. The peaks at $L^{*} \sim 5$ would not have been detected by the Hilmer et al. [2000] study that had measurements only for $L=4.2$ and 6.6 .

[57] In addition, the evidence we have presented for internal acceleration near $L^{*}=5$ is consistent with the findings of a recent study by O'Brien et al. [2003]. The study compared $>1.5 \mathrm{MeV}$ relativistic electron flux to ULF wave power and the occurrence of electron microburst precipitation over a range of radial distances from $L=4.5$ to 6.6. The level of ULF wave power indicates the rate of radial diffusion while microburst occurrence indicates the presence of VLF waves which are a necessary component of many internal acceleration mechanisms. Using 5 years of microburst data and 14 years of ULF wave measurements the study showed a peak in the microburst occurrence at $L \sim=5$ and peak ULF wave power at $L>7$. A comparison of waves and electrons for $\sim 65$ events revealed that relativistic electron flux increases at $L=4.5$ are most 


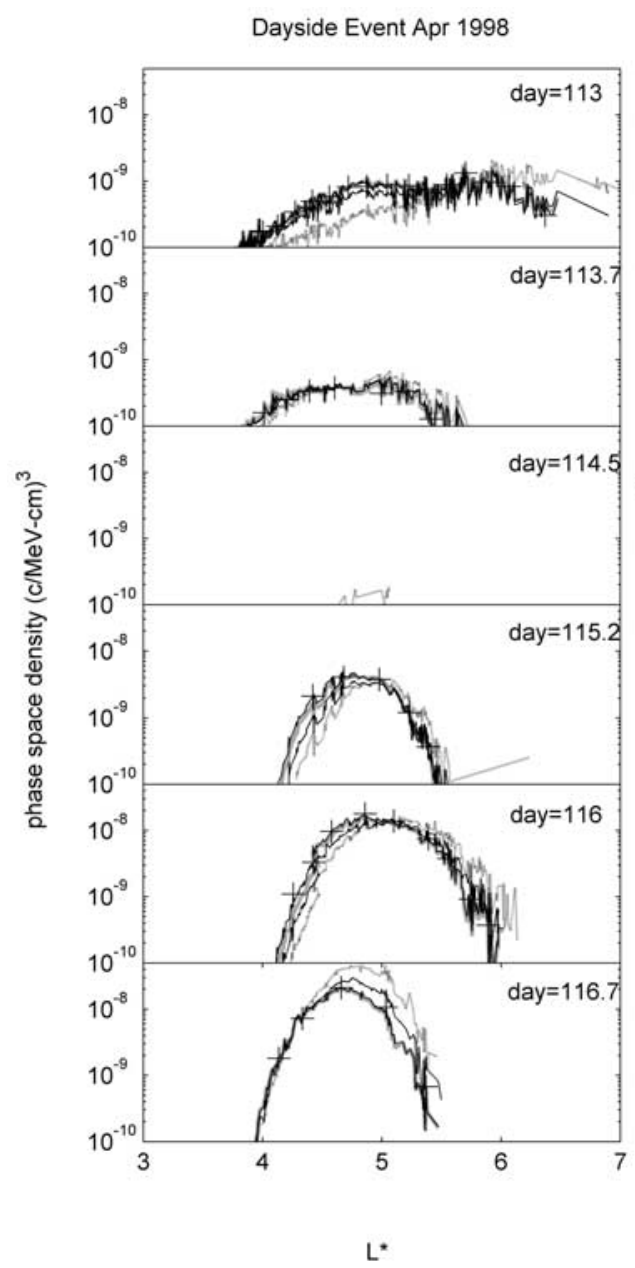

A)

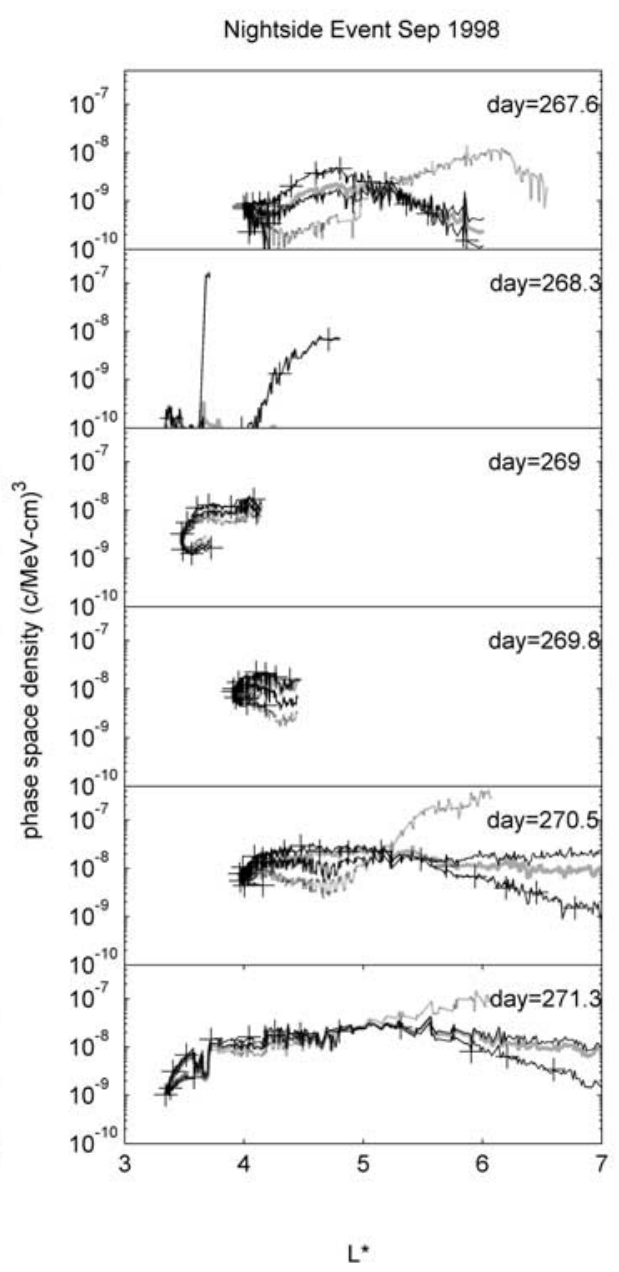

B)

Figure 18. Phase space density calculated using different inputs to the T96 model to demonstrate the sensitivity of the results. Black traces show phase space density calculated using the measured solar wind and Dst values as input to the model. Dashed traces show phase space density calculated using $D s t-40 \mathrm{nT}$ as input, and plus signs show phase space density calculated using Dst $+40 \mathrm{nT}$. Shaded traces show phase space density calculated using the T96 model that minimizes the difference between the local model field and the field measured at Polar. (a) Data from the 23 April event and (b) data from the 24 September event.

closely associated with microburst activity whereas flux increases observed at $L=6.6$ are most closely associated with ULF wave power. The authors conclude that both internal and external source acceleration mechanisms play a role in electron acceleration. They suggest that internal source accelerations mechanisms which include VLF wave interactions dominate at low $L$ and radial diffusion of electrons from an external source outside of geostationary causes electron acceleration at $L=6.6$. An alternative explanation, compatible with the findings of O'Brien et al. [2003] as well as our study, is that electrons are accelerated by an internal acceleration mechanism requiring VLF interaction near $L^{*} \sim 5$, the nominal peak in both phase space density and microburst occurrence. If strong ULF wave power is also present, radial diffusion transports electron outward causing increased electron flux at geosynchronous. For example, outward radial diffusion may contribute to the broadening of the phase space density peak to larger $L^{*}$ values such as seen in Figure 8.

\section{Conclusions}

[58] The analysis of phase space density gradients in this study suggest that relativistic electrons are accelerated primarily by an internal source acceleration mechanism active near $L^{*} \sim 5$. Potential internal source acceleration mechanisms that could explain the observed phase space density gradients include the EMIC/whistler chorus mechanism [Summers et al., 1998], the ULF/VLF recirculation model [Liu et al., 1999], and the ULF transit time damping mechanism [Summers and $M a, 2000]$. The correlation at low $L$ values with increased microbursts shown by $O$ 'Brien et al. [2003] suggest that viable internal acceleration mechanisms must incorporate some type of VLF wave interaction. 
Comparison of Model and Measured Magnetic Field Apr 26,1998 Day of year 116

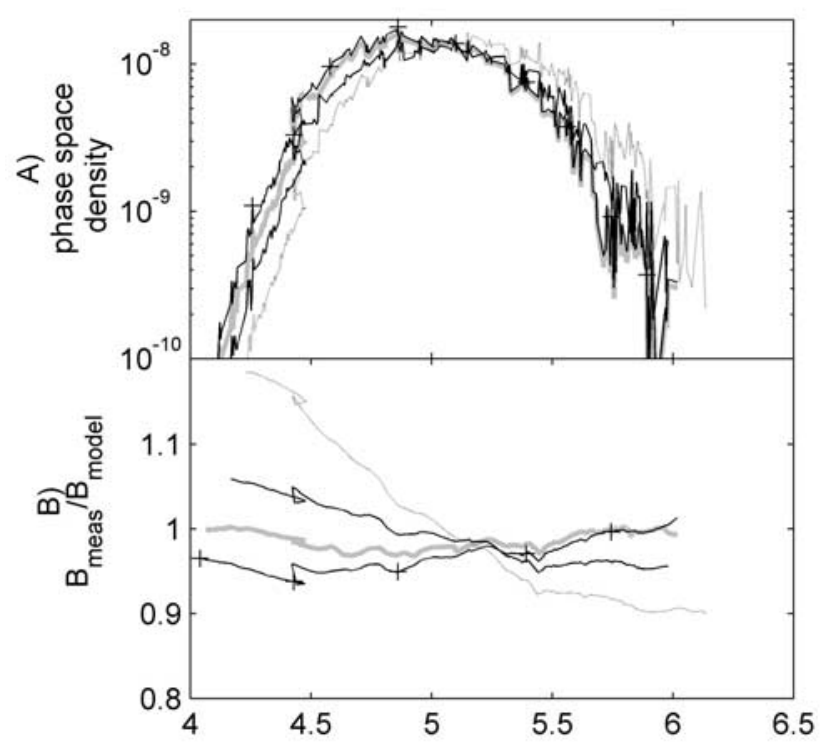

$L^{*}$

Figure 19. (a) Phase space density calculated using different inputs to the T96 model on day 116. (b) A comparison of the measured and model magnetic field during the same time period. The colors and line type are the same as those used in Figure 18.

This requirement makes the relevance of the ULF transit time damping mechanism, uncertain. Although the mechanism does not rely on VLF wave interaction, more analysis is required to determine whether or not it makes any partial contribution to electron acceleration. The three remaining internal acceleration mechanisms that do incorporate VLF wave interaction all violate the electrons' first adiabatic invariant and should make observable changes to electron pitch angle distributions. However, more complete descriptions of the parameters that govern ULF and VLF wave particle interactions such as plasma density, wave power, frequency, $k$ vectors, local time and latitude dependence are required to develop models of pitch angle distributions for comparison with observations. The pieces to the puzzle presented by Van Allen's first radiation belt measurements have narrowed but the complete picture has not yet fully emerged.

\section{Appendix A: Definition of the Adiabatic Invariants}

[59] The many models of relativistic electron acceleration are generally discussed in terms of particle adiabatic invariants. Adiabatic invariants are quantities that describe the general characteristics of the complicated motion of particles in the magnetosphere. The complex motion can be understood more simply by separating it into three types of motion that occur on different timescales: the gyromotion of a particle about a field line, the bounce motion of a particle along a field line and the azimuthal drift of a particle about the Earth [Roederer, 1970; Schulz and Lanzerotti, 1974].
When changes in the magnetic field are slow compared with the period of the motion three quantities corresponding to each type of motion are conserved. These quantities are called the adiabatic invariants and are defined as follows.

[60] The first invariant of the motion is associated with the cyclotron motion of the electron about a field line and is given byFirst invariant

$$
\mu=\frac{p_{\perp}^{2}}{2 m_{0} B}
$$

Here $p_{\perp}$ is the relativistic momentum in the direction perpendicular to the magnetic field, $m_{0}$ is the rest mass of the electron, and $B$ is the field magnitude.

[61] The second invariant corresponds to the bounce motion of a particle along a field line (Figure 7c) and is given by Second invariant

$$
J=\oint p_{\|} d s
$$

where $p_{\|}$is the particle momentum parallel to the magnetic field and $d s$ is the distance a particle travels along the field line. It is convenient to rewrite the second invariant in terms of only the magnetic field geometry by the following manipulation. If no parallel forces act on a particle then momentum is conserved along a bounce path and $J=2 p I$ where $p$ is momentum and $I$ is given as

$$
I=\int_{s_{m}}^{s_{m}^{\prime}}\left[1-\frac{B(s)}{B_{m}}\right]^{\frac{1}{2}} d s
$$

Here $s_{m}$ is the distance of the particle mirror point, $B(s)$ is the field strength at point $s$ and $B_{m}$ is the mirror point magnetic field strength. If the first invariant is conserved then $K$, as defined below, is also conserved.

$$
K=\frac{J}{2 \sqrt{2 m_{0} \mu}}=I \sqrt{B_{m}}=\int_{s_{m}}^{s_{m}^{\prime}}\left[B_{m}-B(s)\right]^{1 / 2} d s
$$

where $m_{0}$ is the rest mass of an electron. Throughout this work we refer to $K$ when speaking of the second invariant.

[62] The third and final invariant corresponds to the drift motion of a particle about the Earth and is given by Third invariant

$$
\Phi=\oint A_{\phi} d l
$$

In this equation $A_{\phi}$ is the magnetic vector potential and $d l$ is the curve along which lies the guiding center drift shell of the electron. Using Stokes theorem the third invariant can be written as

$$
\Phi=\int(\nabla \times A) d S=\int B d S
$$

where $B$ is the magnetic field and $d S$ is area. Therefore, conservation of this invariant requires that an electron always enclose the same amount of magnetic flux as it drifts 
about the Earth. In a dipole field this is equivalent to saying that the electron remains at fixed radial distance. The Roederer $L$ parameter, commonly written as $L^{*}$, is another useful form of the third invariant and is written as

$$
L^{*}=\frac{2 \pi M}{\Phi R_{E}}
$$

where $M$ is the magnetic moment of the Earth's dipole field. The $L^{*}$ parameter is the radial distance to the equatorial location where an electron would be found if all external magnetic fields were slowly turned off leaving only the internal dipole field.

[63] An irreversible increase of relativistic electron flux occurs only when one of the above invariants is violated. Therefore, acceleration mechanisms are described by whether they perturb the gyromotion, bounce motion, or drift motion of an electron violating the first, second, or third adiabatic invariant.

[64] Acknowledgments. This work was supported by a grant from the Aerospace Corporation 4600001602. We thank the Polar Principal Investigators C. T. Russell and J. B. Blake for use of the MFE and HIST data, respectively. We are grateful for the use of the UNILIB code provided via the Internet by the Belgian Institute for Space Aeronomy. We are thankful for the use of the NSSDC OMNIWeb database of solar wind data. We also thank those who contributed data to that database, such as E. C. Stone, R. Lepping, and K. Ogilvie. Finally, we are indebted to J. B. Blake, R. Selesnick, J. Fennell, K. Lorentzen, T. P. O’Brien, T. G. Onsager, R. J. Walker, K. K. Khurana, and S. Thompson, who contributed their knowledge and expertise. UCLA Institute of Geophysics and Planetary Physics publication 5808 .

[65] Lou-Chuang Lee thanks Danny Summers and Scot R. Elkington for their assistance in evaluating this paper.

\section{References}

Albert, J. (2000), Gyroresonant interaction of radiation belt particles with a monochromatic electromagnetic wave, J. Geophys. Res., 105, 21,191.

Barton, C. E. (1997), International geomagnetic reference field: The seventh generation, J. Geomagn. Geoelectr., 49, 123-148.

Blake, J., et al. (1995), CEPPAD: Comprehensive Energetic Particle and Pitch Angle Distribution Experiment on Polar, Space Sci. Rev., 71, 531.

Brautigam, D. H., and J. M. Albert (2000), Radial diffusion analysis of outer radiation belt electrons during the October 9, 1990, magnetic storm, J. Geophys. Res., 105, 291.

Contos, A. (1997), A complete description of the high sensitivity telescope (HIST) onboard the Polar satellite, M.S. thesis, Boston Univ., Boston, Mass.

Elkington, S. R., M. K. Hudson, and A. A. Chan (1999), Acceleration of relativistic electrons via drift resonance intraction with toroidal-mode Pc5 ULF oscillations, Geophys. Res. Lett., 26, 3273.

Elkington, S. R., M. K. Hudson, and A. A. Chan (2003), Resonant acceleration and diffusion of outer zone electrons in an asymmetric geomagnetic field, J. Geophys. Res., 108(A3), 1116, doi:10.1029/2001JA009202.

Falthammer, C.-G. (1965), Effects of time-dependent electric fields on geomagnetically trapped radiation, J. Geophys. Res., 70, 2503.

Falthammer, C.-G. (1966), On the transport of trapped particles in the outer magnetosphere, J. Geophys. Res., 71, 1487.

Friedel, R. H. W., G. D. Reeves, and T. Obara (2002), Relativistic electron dynamics in the inner magnetosphere-a review, J. Atmos. Solar Terr. Phys., 64, 265.
Green, J. C. (2002), Testing relativistic electron acceleration mechanisms, Ph.D. thesis, Univ. of Calif., Los Angeles.

Green, J. C., and M. G. Kivelson (2001), A tale of two theories: How the adiabatic response and ULF waves affect relativistic electrons, J. Geophys. Res., 106, 25,777.

Hilmer, R. V., G. P. Ginet, and T. E. Cayton (2000), Enhancement of equatorial energetic electron fluxes near $L=4.2$ as a result of high speed solar wind streams, J. Geophys. Res., 105, 23,311.

Hudson, M. K., S. R. Elkington, J. G. Lyon, and C. C. Goodrich (2000), Increase in relativistic electron flux in the inner magnetosphere: ULF wave mode structure, Adv. Space Res., 25, 2327.

Kim, H.-J., A. Chan, R. A. Wolf, and J. Birn (2000), Can substorms produce relativistic outer belt electrons?, J. Geophys. Res., 105, 7721.

Li, X., D. N. Baker, M. G. D. Reeves, and D. Larson (2001), Quantitative prediction of radiation belt electrons at geostationary orbit on the basis of solar wind measurements, Geophys. Res. Lett., 28, 1887.

Liu, W., G. Rostocker, and D. Baker (1999), Internal acceleration of relativistic electrons by large amplitude ULF pulsations, J. Geophys. Res., 104, 17,391.

Lorentzen, K. R., M. D. Looper, and J. B. Blake (2001), Relativistic electron microbursts during the GEM storms, Geophys. Res. Lett., 28, 2573.

O’Brien, T. P., K. R. Lorentzen, I. R. Mann, N. P. Meredith, J. B. Blake, J. F. Fennell, M. D. Looper, D. K. Milling, and R. R. Anderson (2003), Energization of relativistic electrons in the presence of ULF power na $\mathrm{dMeV}$ microbursts: Evidence for dual ULF and VLF acceleration, J. Geophys. Res., 108(A8), 1329, doi:10.1029/2002JA009784.

Olson, W. P. (1974), A model of the distributed magnetospheric currents, J. Geophys. Res., 79, 3731.

Press, W. H., B. P. Flannery, S. A. Teukolsky, and W. T. Vetterling (1988), Numerical Recipes in $C$, Cambridge Univ. Press, New York.

Roederer, J. G. (1970), Dynamics of Geomagnetically Trapped Radiation, Cambridge Univ. Press, New York.

Russell, C. T., R. C. Snare, J. D. Means, D. Pierc, D. Dearborn, M. Larson, G. Barr, and G. Le (1995), The GGS/Polar magnetic fields investigation, Space Sci. Rev., 71, 563.

Schulz, M., and L. Lanzerotti (1974), Particle Diffusion in the Radiation Belts, vol. 7, Springer-Verlag, New York.

Selesnick, R., and J. B. Blake (2000), On the source location of radiation belt electrons, J. Geophys. Res., 105, 2607.

Selesnick, R., and J. B. Blake (2002), Relativistic electron drift shell splitting, J. Geophys. Res., 107, 1265, doi:10.1029/2001JA009179.

Shabansky, V. P. (1971), Some processes in the magnetosphere, Space Sci. Rev., 12, 299.

Summers, D., and C.-Y. Ma (2000), Rapid acceleration of electrons in the magnetosphere by fast mode MHD waves, J. Geophys. Res., 105, 15,887.

Summers, D., R. M. Thorne, and F. Xiao (1998), Relativistic theory of wave particle resonant diffusion with application to electron acceleration in the magnetosphere, J. Geophys. Res., 103, 20,487.

Tsyganenko, N. A. (1989), A magnetospheric magnetic field model with a warped tail current, Planet. Space Sci., 37, 5.

Tsyganenko, N., and D. P. Stern (1996), Modeling the global magnetic field of the large scale birkeland current systems, J. Geophys. Res., 101, 187.

Van Allen, J. A., and L. A. Frank (1959), Radiation Around the Earth to a radial distance of 107,400 km, Nature, 183, 430.

Van Allen, J. A., C. E. McIlwain, and G. H. Ludwig (1959), Radiation observations with satellite 1958 Epsilon, J. Geophys. Res., 64, 271.

Walt, M. (1994), Introduction to Geomagnetically Trapped Radiation, Cambridge Univ. Press, New York.

J. C. Green, Laboratory for Atmospheric and Space Physics, University of Colorado, 1234 Innovation Dr., Boulder, CO 80303, USA. (janet. green@lasp.colorado.edu)

M. G. Kivelson, Institute of Geophysics and Planetary Physics and Department of Earth and Space Sciences, University of California, Los Angeles, CA 90095-1567, USA. 

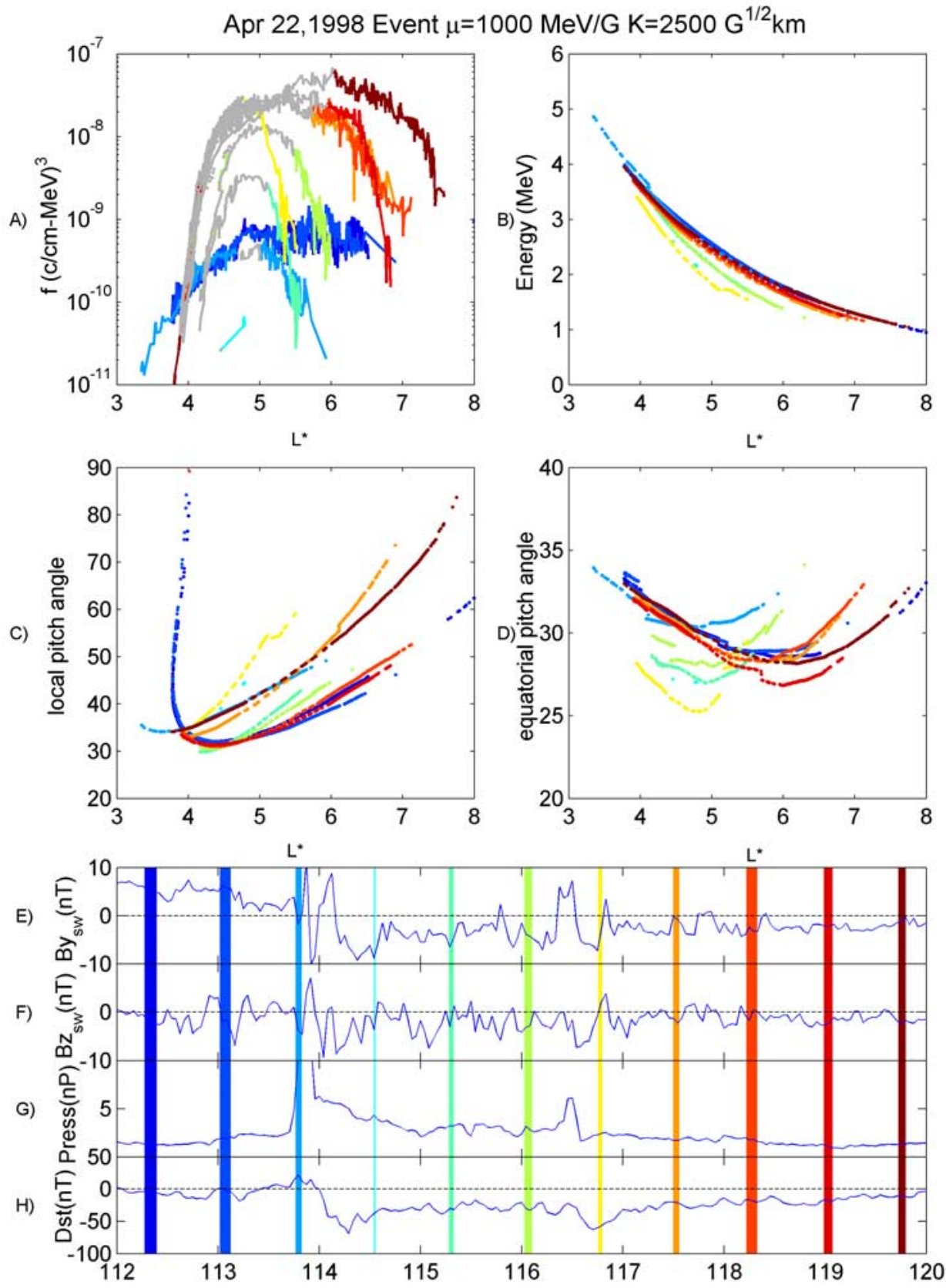

Day of year 1998

Figure 8. Example of an acceleration event that relies predominantly on data taken from the dayside portion of the Polar orbit and shows increasing phase space density peaks at intermediate $L^{*}$ indicative of internal acceleration. (a) Phase space density of electrons in units of $(\mathrm{c} / \mathrm{cm}-\mathrm{MeV})^{3}$ where $\mathrm{c}$ is the speed of light for $\mu=1000 \mathrm{MeV} / \mathrm{G}$ and $K=2500 \mathrm{G}^{1 / 2} \mathrm{~km}$ from 23 to 29 April. The data are plotted versus $L^{*}$ and are color coded in time with each color corresponding to a different orbit. (b) The energy (MeV) of electrons used in the calculation of phase space density. (c) The local pitch angle (degrees) of electrons used in the calculation and (d) the equatorial pitch angle (degrees) of the electrons. (e) The $B_{y}$ (nT) component and (f) the $B_{z}(\mathrm{nT})$ component of the solar wind in GSM coordinates. (g) The dynamic pressure (nP) and (h) Dst (nT). Vertical bars mark the times during the Polar orbit that the radiation belts were encountered. These bars are color coded to match the data plotted in Figures 8a-8d. 

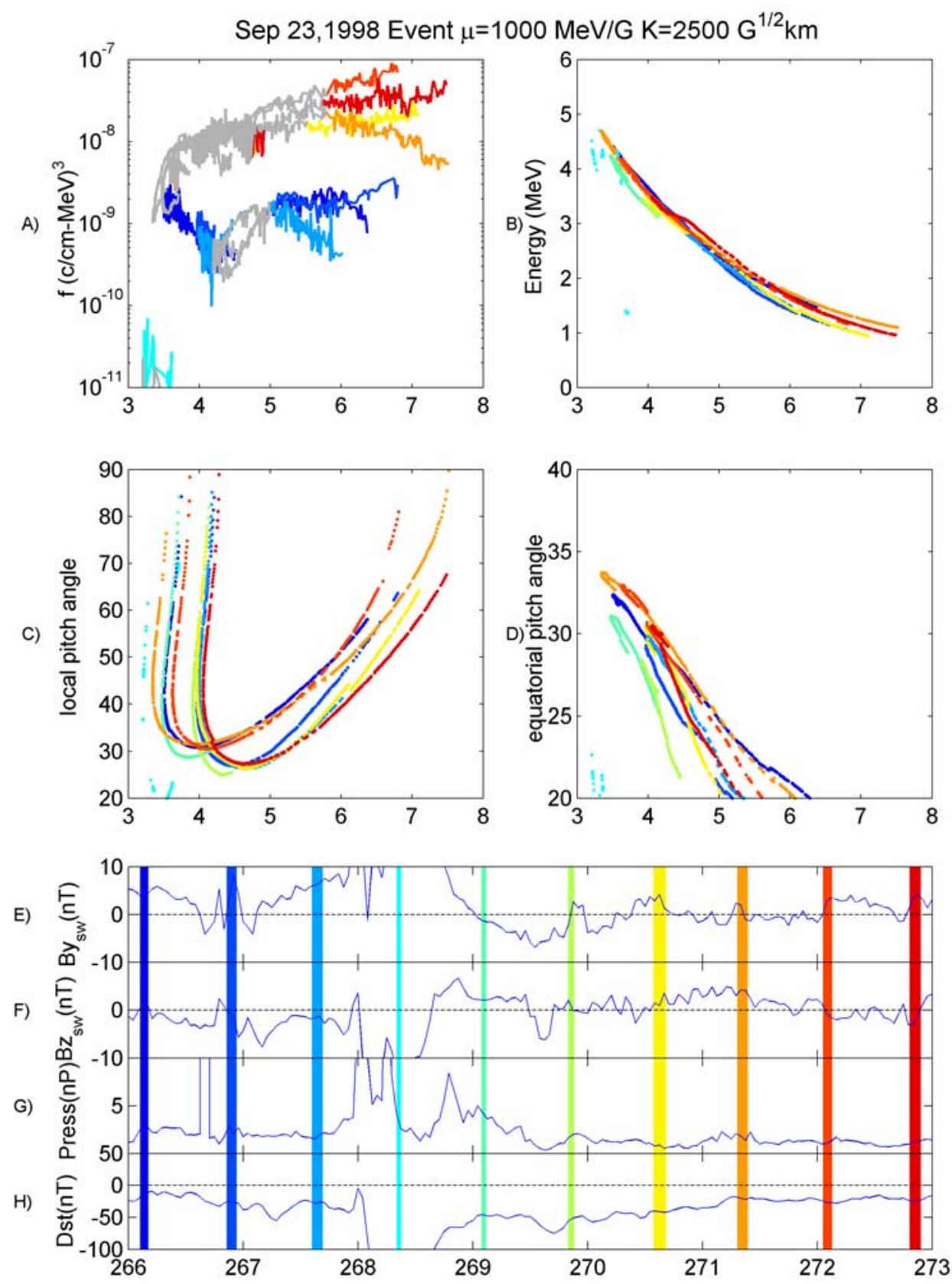

Day of year 1998

Figure 12. Example of an acceleration event that relies predominantly on data taken from the nightside portion of the Polar orbit and shows phase space density gradients indicative of external acceleration. The format is the same as for Figure 8 . 


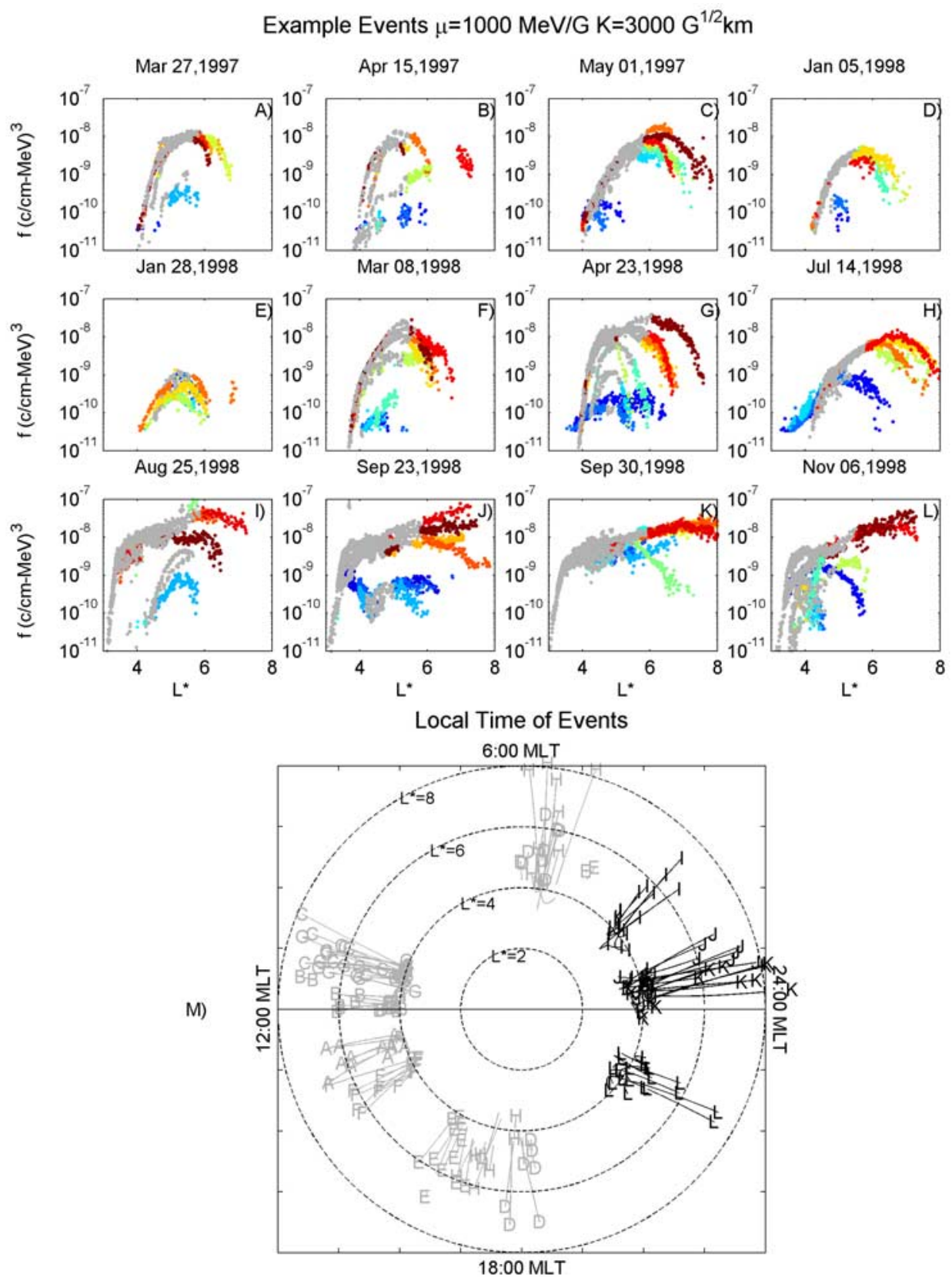

Figure 15. Demonstration of the local time dependence of phase space density versus $L^{*}$ profiles. (a)-(1) Phase space density $(\mathrm{c} / \mathrm{cm}-\mathrm{MeV})^{3}$ versus $L^{*}$ for 12 events from January 1997 to January 1999. (m) The local time of each event. Each trace in Figure $15 \mathrm{~m}$ is labeled with a letter corresponding to the event plotted in Figures 15a-15l. 